\title{
Geographical Distribution of Diurnal and Semidiurnal Parametric Subharmonic Instability in a Global Ocean Circulation Model
}

\author{
Joseph K. Ansong, ${ }^{\mathrm{a}, \mathrm{b}}$ Brian K. Arbic, ${ }^{\mathrm{a}}$ Harper L. Simmons, ${ }^{\mathrm{c}}$ Matthew H. Alford, ${ }^{\mathrm{d}, \mathrm{e}}$ \\ MAARTEn C. BuiJsman, ${ }^{\mathrm{f}}$ PATRICK G. Timko, ${ }^{\mathrm{g}}$ JAMES G. RiCHMAN, ${ }^{\mathrm{h}}$ JAY F. SHRIVER, ${ }^{\mathrm{i}}$ \\ AND ALAN J. WALLCRAFT ${ }^{\text {h }}$ \\ ${ }^{a}$ Department of Earth and Environmental Sciences, University of Michigan, Ann Arbor, Michigan \\ ${ }^{\mathrm{b}}$ Department of Mathematics, University of Ghana, Legon, Accra, Ghana \\ ${ }^{\mathrm{c}}$ College of Fisheries and Ocean Sciences, University of Alaska Fairbanks, Fairbanks, Alaska \\ ${ }^{\mathrm{d}}$ Applied Physics Laboratory, University of Washington, Seattle, Washington \\ e School of Oceanography, University of Washington, Seattle, Washington \\ ${ }^{\mathrm{f}}$ Division of Marine Science, University of Southern Mississippi, Stennis Space Center, Mississippi \\ ${ }^{\mathrm{g}}$ Centre for Applied Marine Sciences, Bangor University, Anglesey, United Kingdom \\ ${ }^{\text {h }}$ Center for Ocean-Atmospheric Prediction Studies, Florida State University, Tallahassee, Florida \\ i Ocean Dynamics and Prediction Branch, Naval Research Laboratory, Stennis Space Center, Mississippi
}

(Manuscript received 19 August 2017, in final form 2 February 2018)

\begin{abstract}
The evidence for, baroclinic energetics of, and geographic distribution of parametric subharmonic instability (PSI) arising from both diurnal and semidiurnal tides in a global ocean general circulation model is investigated using $1 / 12.5^{\circ}$ and $1 / 25^{\circ}$ simulations that are forced by both atmospheric analysis fields and the astronomical tidal potential. The paper examines whether PSI occurs in the model, and whether it accounts for a significant fraction of the tidal baroclinic energy loss. Using energy transfer calculations and bispectral analyses, evidence is found for PSI around the critical latitudes of the tides. The intensity of both diurnal and semidiurnal PSI in the simulations is greatest in the upper ocean, consistent with previous results from idealized simulations, and quickly drops off about $5^{\circ}$ from the critical latitudes. The sign of energy transfer depends on location; the transfer is positive (from the tides to subharmonic waves) in some locations and negative in others. The net globally integrated energy transfer is positive in all simulations and is $0.5 \%-10 \%$ of the amount of energy required to close the baroclinic energy budget in the model. The net amount of energy transfer is about an order of magnitude larger in the $1 / 25^{\circ}$ semidiurnal simulation than the $1 / 12.5^{\circ}$ one, implying the dependence of the rate of energy transfer on model resolution.
\end{abstract}

\section{Introduction}

Internal tides are generated by barotropic tidal flow over rough topography in a stratified fluid. After generation, vertical low-mode internal tides may radiate into the interior of the ocean or break and mix in the vicinity of rough topography. Internal tides that escape away from their sources do not travel indefinitely since they are bound by their turning latitudes (the latitude at which their frequency is equal to the local inertial frequency; Hendershott 1973; Wunsch and Gill 1976).

Corresponding author: Joseph K. Ansong, jkansong@umich.edu, jkansong@ug.edu.gh
There are several unanswered questions about the fate of low-mode internal tides radiating away from topographic sources:

1) What percentage of converted barotropic-to-baroclinic energy eventually goes into mixing the ocean? Mixing in the deep ocean, now thought to be primarily due to the baroclinic tides, wind-generated near-inertial waves (NIWs), and the wind-driven general circulation, has been argued to exert a strong influence on the stratification and general circulation of the ocean (Munk and Wunsch 1998; St. Laurent and Simmons 2006; MacKinnon et al. 2017).

2) What are the dynamic processes befalling internal tides before they finally break? 
3) What is the best way of parameterizing dissipation of internal waves in global ocean models?

Recent progress on the questions above and on other related physical oceanography problems has been reviewed by a Climate Process Team on internal-wave-driven ocean mixing (MacKinnon et al. 2017).

Parametric subharmonic instability (PSI) is one of several potential processes responsible for removing energy from internal tides on their journey from topographic sources. PSI is a particular resonant wave-wave interaction in which a primary wave (the internal tide in this case) exchanges energy with daughter waves with smaller vertical scales and with nearly half the frequency of the primary wave (McComas and Bretherton 1977). Observational studies (Alford et al. 2007; Qiu et al. 2012; MacKinnon et al. 2013a,b; Sun and Pinkel 2012, 2013; Alford et al. 2017), studies utilizing idealized numerical models (Hibiya et al. 1998, 2002; MacKinnon and Winters 2005; Furuichi et al. 2005; Gerkema et al. 2006; Carter and Gregg 2006; Hazewinkel and Winters 2011; Gayen and Sarkar 2013; Richet et al. 2017), and realistic global numerical models (Simmons 2008) continue to implicate PSI for shaping the internal wave dynamics. Innovative laboratory experiments have also been designed to study PSI (see Sutherland 2013, and references therein; Bourget et al. 2014). The idealized numerical study of MacKinnon and Winters (2005) found a significant loss of energy from the $\mathrm{M}_{2}$ internal tide at its critical latitude (CL) of $28.8^{\circ}$. Subsequent observational studies found that the energy loss at $28.8^{\circ}$ was not as catastrophic as predicted by the idealized study. Alford et al. (2007) suggest that one possible reason for this discrepancy is the presence of higher-mode waves in the real ocean. These waves move in different directions (see Zhao et al. 2010), thus detuning the perfect phase locking that resulted in the exponential PSI growth in MacKinnon and Winters (2005).

An idealized numerical study by Hazewinkel and Winters (2011) built upon the simulations of MacKinnon and Winters (2005) by incorporating a vertically nonuniform density stratification, the planetary beta effect, and higher vertical resolution into their simulations. They observed PSI to occur in the upper ocean with subsequent propagation of near-inertial oscillations (NIOs) into the deep ocean. Importantly, they found that the reduction of baroclinic energy flux due to PSI is sensitive to eddy viscosity. Using a vertical eddy viscosity of $5 \times 10^{-5} \mathrm{~m}^{2} \mathrm{~s}^{-1}$, they found a flux reduction of about $15 \%$, consistent with the observations of Alford et al. (2007) for beams crossing the critical latitude around Hawaii. Recent analyses of observational data by Alford et al. (2017) further implicate PSI for heightened near-inertial shear in the upper ocean around the North Pacific CL. Using idealized numerical simulations, Richet et al. (2017) find that the presence of a mean flow removes the tidal dissipation peak at the PSI critical latitude, and they reported two weak peaks away from the CL. They attribute the sensitivity of PSI to the mean current that Doppler shifts the frequency of the primary internal tide.

Simmons (2008) demonstrated that the PSI seen at critical latitudes in the idealized models run by MacKinnon and Winters (2005) can also be seen in a realistic global internal tide model. However, the model used in Simmons (2008) had several simplifications compared to the actual ocean. These include the use of a horizontally uniform stratification, the inclusion of only one tidal constituent (the $\mathrm{M}_{2}$ semidiurnal tide), and the lack of atmospheric forcing. The results presented here come from three separate simulations set up to mimic realistic oceanic conditions through

1) forcing of the model with the three major semidiurnal tides in two simulations and with the three major diurnal tides in a third,

2) inclusion of atmospheric forcing, and

3) the resulting horizontally varying stratification.

The inclusion of atmospheric forcing ensures the presence of a vigorous mesoscale eddy field as well as the generation of NIWs. Both eddies and NIOs may interact with low-mode internal tides, potentially detuning them and altering the PSI behaviors. The inclusion of different tidal constituents is also important because the study by Hazewinkel and Winters (2011) found that the growth rate of PSI is influenced by the spring-neap cycle. In addition, it is of interest to examine whether diurnal PSI can be detected in a global ocean model, because observational studies (Alford 2008; Xie et al. 2016) and idealized numerical studies (Simmons 2008) both suggest its occurrence.

\section{The HYCOM model and methodology}

The simulations in this study were performed with the Hybrid Coordinate Ocean Model (HYCOM; Bleck 2002; Halliwell 2004; Chassignet et al. 2009), which is in use by the U.S. Navy as an operational model (Metzger et al. 2014). The simulations used here, however, are run in forward (non-data-assimilative) mode. Two simulations are forced by the three largest semidiurnal tidal constituents $\left(\mathrm{M}_{2}, \mathrm{~S}_{2}, \mathrm{~N}_{2}\right)$ at horizontal resolutions of $1 / 12.5^{\circ}$ and $1 / 25^{\circ}$ at the equator. A third simulation is forced by the three largest diurnal constituents $\left(\mathrm{Q}_{1}, \mathrm{O}_{1}, \mathrm{~K}_{1}\right)$ at a horizontal resolution of $1 / 12.5^{\circ}$ at the equator. The model simulations use K-profile parameterization 
(KPP; Large et al. 1994) as their mixed layer submodel. We use a critical bulk Richardson number of 0.25 . For the shear instability term we set the maximum gradient Richardson number to 0.7 with a cubic profile from 0 to 0.7 and a maximum value of $50 \times 10^{-4} \mathrm{~m}^{2} \mathrm{~s}^{-1}$ for viscosity and diffusivity. The vertical eddy diffusivity for unresolved background internal wave shear is prescribed as $1.0 \times 10^{-5} \mathrm{~m}^{2} \mathrm{~s}^{-1}$ while the vertical eddy viscosity due to unresolved background internal waves is $3.0 \times 10^{-5} \mathrm{~m}^{2} \mathrm{~s}^{-1}$ (Wallcraft et al. 2009), a value comparable to that used in Hazewinkel and Winters (2011). The model has 41 hybrid vertical coordinate surfaces with potential density referenced to $2000 \mathrm{~m}$ and atmospheric forcing from the Navy Global Environmental Model (NAVGEM; Hogan et al. 2014).

We employ the parameterized topographic wave drag scheme of Jayne and St Laurent (2001). Because global models are not able to resolve the breaking of internal waves, we use a parameterized wave drag acting on the bottom flow to represent the generation and breaking of unresolved high vertical modes by flow over topography (Arbic et al. 2004, 2010). Ansong et al. (2015) showed that the barotropic and low-mode baroclinic tides in simulations forced simultaneously by tides and atmospheric fields compare more closely to satellite altimeter observations when a parameterized internal wave drag is applied to the bottom flow. Buijsman et al. (2016) showed that about $50 \%$ of the internal wave dissipation can be attributed to bottom drag in $1 / 12.5^{\circ} \mathrm{HYCOM}$. Because of storage limitations, the recorded hourly global three-dimensional model output is held to 60 days for the runs at $1 / 12.5^{\circ}$ resolution and to 30 days for the $1 / 25^{\circ}$ simulation. This amount of data requires about 48 terabytes of disk space from all simulations. Other previous papers (Arbic et al. 2010, 2012; Timko et al. 2012, 2013; Richman et al. 2012; Shriver et al. 2012, 2014; Ansong et al. 2015; Buijsman et al. 2015, 2016) can be consulted for detailed discussions of the parameterized topographic internal wave drag, self-attraction and loading, and other important details in our tideresolving HYCOM simulations. Shriver et al. (2012) demonstrated that the amplitudes of the barotropic and internal tide sea surface elevations in HYCOM compare well to altimeter-constrained models and along-track altimeters, respectively. The tidal currents in HYCOM have been compared to archived current meter records spanning about 40 years (Timko et al. 2012, 2013). On average over 5000 locations, Timko et al. (2013) find that the kinetic energy of the $\mathrm{M}_{2}$ tide is within a factor of 1.3 of observations. Other model-data comparisons performed with HYCOM include Müller et al. (2015), who compared the internal gravity wave (IGW) kinetic energy (KE) frequency spectral densities to current

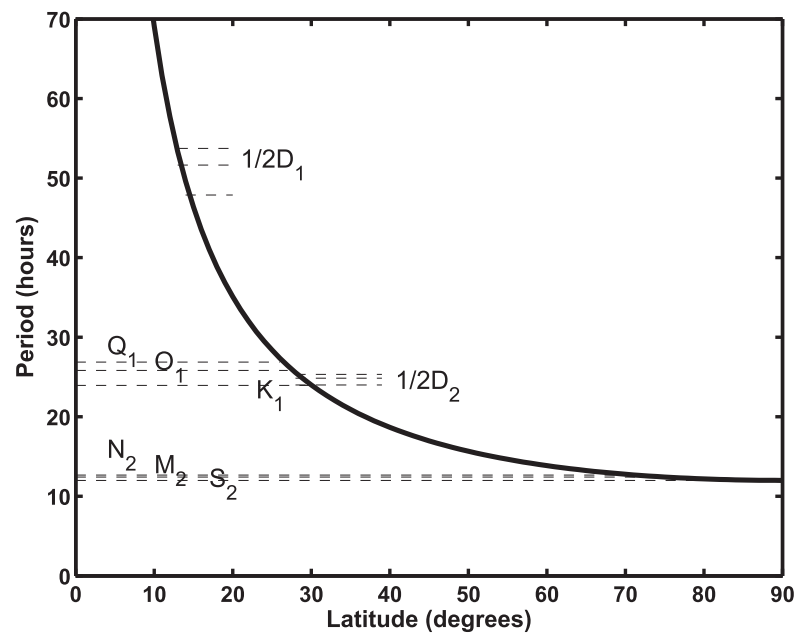

FIG. 1. Periods of the diurnal $\left(\mathrm{K}_{1}, \mathrm{O}_{1}, \mathrm{Q}_{1} ; \mathrm{D}_{1}\right.$ collectively) and semidiurnal $\left(\mathrm{M}_{2}, \mathrm{~S}_{2}, \mathrm{~N}_{2} ; \mathrm{D}_{2}\right.$ collectively) tides as well as periods of their half-frequency subharmonics $\left[(1 / 2) \mathrm{D}_{1}\right.$ and $(1 / 2) \mathrm{D}_{2}$, respectively]. The solid curve shows the local period of inertial oscillations $f$ as a function of latitude. Lines to the left of $f$ are for $\mathrm{D}_{2}$ and $\mathrm{D}_{1}$; those to the right are for $(1 / 2) \mathrm{D}_{2}$ and $(1 / 2) \mathrm{D}_{1}$.

meter spectral densities; Ansong et al. (2017), who compared the baroclinic tidal energy fluxes to fluxes computed from current meter records; and Savage et al. (2017), who compared the dynamic height frequency spectral densities with in situ depth profiling instruments.

Figure 1 depicts the local period of inertial oscillations versus latitude. On the same plot we show the periods of the diurnal and semidiurnal tides. We note that this is a schematic that is only used to help in interpretation; in the real ocean the tidal periods could be Doppler shifted by other motions. The CLs of the three diurnal tidal constituents $\left(\mathrm{Q}_{1}, \mathrm{O}_{1}, \mathrm{~K}_{1}\right)$ - the location where half their frequency equals the local inertial frequency-are $12.9^{\circ}$, $13.4^{\circ}$, and $14.5^{\circ}$, respectively. The CLs of the three semidiurnal constituents $\left(\mathrm{N}_{2}, \mathrm{M}_{2}, \mathrm{~S}_{2}\right)$ are $28.2^{\circ}, 28.8^{\circ}$, and $29.9^{\circ}$, respectively. Because the semidiurnal CLs occur near the turning latitudes of the diurnal tides, spectral separation is impossible without very long time series data. This is the main reason that we forced the model with the diurnal and semidiurnal tides separately, in contrast to the real ocean where the tidal constituents occur concurrently. The semidiurnal and diurnal signals are obtained by a Butterworth filter that bandpasses between 1.55 and $2.32 \mathrm{cpd}$ and 0.80 and $1.20 \mathrm{cpd}$, respectively. Their subharmonics are obtained by a bandpass between 0.80 and $1.20 \mathrm{cpd}$ and 0.40 and $0.60 \mathrm{cpd}$, respectively. We use HYCOM12S and HYCOM12D to represent the HYCOM simulations forced by the semidiurnal and diurnal tides, respectively, at $1 / 12.5^{\circ}$ resolution, and HYCOM25S to represent the $1 / 25^{\circ}$ simulation. The Rayleigh criterion that the differences 
between resolved frequencies must be greater than the inverse of the data record length implies that the local near-inertial oscillations cannot be separated from the semidiurnal subharmonic waves between about latitudes $\pm 27.1^{\circ}$ and $30.6^{\circ}$ for $30-60$ days.

\section{Theory}

\section{a. Baroclinic energetics}

The energy balance analyses of the total barotropic and baroclinic semidiurnal tides in a HYCOM simulation run at $1 / 12.5^{\circ}$ horizontal resolution are given in Buijsman et al. (2016). In this study, we analyze the baroclinic energy balance following the same approach as discussed in Buijsman et al. (2016), namely,

$$
\{C\}=\{\nabla \cdot \mathbf{F}\}+\left\{D_{l}\right\},
$$

where curly brackets indicate area and time averages, $C$ is the barotropic to baroclinic conversion rate, $\mathbf{F}$ is the baroclinic energy flux vector, and $D_{l}$ is the energy dissipation. Time-averaged and domain-integrated values of the terms in Eq. (1) represent the amount of energy converted from the barotropic to the baroclinic tide (conversion); positive flux divergence represents generation of baroclinic energy while negative divergence indicates dissipation or energy conversion to barotropic tides. The dissipation term represents all processes removing energy from the internal tide including wave-wave interactions and the parameterized topographic internal wave drag (see section 2). The energy dissipation term is further separated into the dissipation due to the topographic wave $\operatorname{drag} D_{w l}$ and quadratic bottom friction $D_{b l}$, such that (Buijsman et al. 2016)

$$
\left\{D_{l}\right\}=\left\{D_{w l}\right\}+\left\{D_{b l}\right\}+\left\{R_{l}\right\},
$$

where $R_{l}$ is a residual term composed of discretization errors, viscous dissipation, and energy loss due to nonlinear wave-wave interactions of the tide and other motions. As in Buijsman et al. (2016), the wave drag and quadratic bottom friction terms are computed using the linear separation technique of Kang and Fringer (2012), to arrive at

$$
\begin{aligned}
\left\{D_{b l}\right\} & =\left\langle\rho_{0} C_{D}\left|\mathbf{u}_{a}\right|\left(u_{a} u_{l a}+v_{a} v_{l a}\right)\right\rangle, \quad \text { and } \\
\left\{D_{w l}\right\} & =\left\langle\rho_{0} \mathbb{C}\left(u_{\alpha} u_{l \alpha}+v_{\alpha} v_{l \alpha}\right)\right\rangle,
\end{aligned}
$$

where the bottom drag coefficient is $C_{D}=0.0025$; subscripts $a$ and $\alpha$ indicate averaging over the bottom 10 and $500 \mathrm{~m}$, respectively; $\rho_{0}$ is the average density of seawater, and $\mathbb{C}$ is a linear wave drag coefficient with units of meters per second. In Eqs. (3) and (4), $(u, v)$ are horizontal velocity components in the zonal and meridional directions, respectively, and velocities in parentheses are bandpassed, whereas the term $\left|\mathbf{u}_{a}\right|$ is the total unfiltered velocities. The variables $u_{l a}$ and $v_{l a}$ are the zonal $(u)$ and meridional $(v)$ velocity components, respectively, of the low-mode tide averaged over the bottom $10 \mathrm{~m}$.

\section{b. PSI energy transfer}

We next present the basic theory of energy transfer to subharmonic signals from the low-mode tide, following previous studies (e.g., Frajka-Williams et al. 2006; MacKinnon et al. 2013b; Sun and Pinkel 2013). In theory the wave vectors and frequencies $\left(\mathbf{k}_{1}, \omega_{1}\right),\left(\mathbf{k}_{2}, \omega_{2}\right)$, $\left(\mathbf{k}_{3}, \omega_{3}\right)$, of the waves in a PSI triad interaction satisfy the resonant conditions

$$
\omega_{1}+\omega_{2}=\omega_{3}, \quad \mathbf{k}_{1}+\mathbf{k}_{2}=\mathbf{k}_{3},
$$

with $\omega_{1} \approx \omega_{2}=\omega_{3} / 2$. The variables in Eq. (5) with subscripts 1 and 2 refer to the daughter waves of PSI, and the third wave is the parent wave. Consider the momentum equations for the inviscid Boussinesq system

$$
\frac{\partial \mathbf{u}}{\partial t}+\mathbf{u} \cdot \nabla \mathbf{u}=\mathrm{OT}
$$

where OT refers to other terms not considered in our analysis, and let

$$
\mathbf{u}=\mathbf{u}_{1}+\mathbf{u}_{2}+\mathbf{u}_{3} .
$$

The velocities may be expressed in terms of Fourier coefficients; for example, $\mathbf{u}_{1}=\hat{\mathbf{u}}_{1} e^{i\left(\mathbf{k}_{1} \cdot \mathbf{x}-\omega_{1} t\right)}+$ c.c., where c.c. refers to the complex conjugate. The energy equation for one of the daughter waves, say $\mathbf{u}_{1}$, can be derived by first substituting Eq. (7) into Eq. (6) and multiplying through by $\mathbf{u}_{1}^{*}$ (the complex conjugate of $\mathbf{u}_{1}$ ). By averaging over large time and space scales, the oscillatory terms vanish, resulting in (Sun and Pinkel 2013)

$$
\frac{\partial E_{1}}{\partial t}+\mathbf{u}_{1}^{*} \cdot\left(\mathbf{u}_{1}+\mathbf{u}_{2}+\mathbf{u}_{3}\right) \cdot \nabla\left(\mathbf{u}_{1}+\mathbf{u}_{2}+\mathbf{u}_{3}\right)=0,
$$

where $E_{1}=(1 / 2)\left\|\mathbf{u}_{1}\right\|^{2}=(1 / 2)\left(u_{1}^{*} u_{1}+v_{1}^{*} v_{1}\right)$ and where we omit the symbol in the above expression and subsequent equations. The terms $\mathbf{u}_{1}^{*} \cdot\left(\mathbf{u}_{2} \cdot \nabla \mathbf{u}_{3}\right)$ and $\mathbf{u}_{1}^{*} \cdot\left(\mathbf{u}_{3} \cdot \nabla \mathbf{u}_{2}\right)$ control the rate of energy transfer since they drive $\mathbf{u}_{1}$ resonantly if the waves satisfy the resonant conditions. Thus, Eq. (8) reduces to the energy equation for $\mathbf{u}_{1}$ :

$$
\frac{\partial E_{1}}{\partial t}=\left[-\mathbf{u}_{1}^{*} \cdot\left(\mathbf{u}_{2} \cdot \nabla \mathbf{u}_{3}\right)-\mathbf{u}_{1}^{*} \cdot\left(\mathbf{u}_{3} \cdot \nabla \mathbf{u}_{2}\right)\right]+\text { c. c. }
$$


The equation governing energy transfers to $\mathbf{u}_{2}$ can be derived in a similar manner. Previous studies (e.g., MacKinnon and Winters 2005; Young et al. 2008) suggest that, near the critical latitude, the term $T=$ $-\mathbf{u}_{1}^{*} \cdot\left(\mathbf{u}_{2} \cdot \nabla \mathbf{u}_{3}\right)$ is the primary driver of PSI rather than the term $-\mathbf{u}_{1}^{*} \cdot\left(\mathbf{u}_{3} \cdot \nabla \mathbf{u}_{2}\right)$. We will show that, indeed, the term $-\mathbf{u}_{1}^{*} \cdot\left(\mathbf{u}_{3} \cdot \nabla \mathbf{u}_{2}\right)$ is negligible everywhere in the model. We will compute energy transfers from term $T$, which may be written in expanded form as

$$
T=-u_{1}^{*}\left(u_{2} \frac{\partial u_{3}}{\partial x}+v_{2} \frac{\partial u_{3}}{\partial y}\right)-v_{1}^{*}\left(u_{2} \frac{\partial v_{3}}{\partial x}+v_{2} \frac{\partial v_{3}}{\partial y}\right) .
$$

In the equations above we have ignored the partial derivatives with respect to $z$ because, close to the critical latitudes where the PSI daughter waves have enhanced energy, the waves have vanishing vertical velocities and displacements (MacKinnon et al. 2013b), resulting in the relatively simpler expression in Eq. (10). To compute the energy transfer terms, we first employ bandpass filtering to obtain the horizontal velocities of the semidiurnal tide. The vertical wavenumber resonant condition in Eq. (5) implies that the daughter waves in a PSI triad interaction with smaller vertical scales than the tide have oppositely signed vertical wavenumbers. Thus, to obtain the subharmonic signals at each grid point, we first bandpass for velocities with half the frequency of the tide, and then use rotary analysis to separate the signals into clockwise $(\mathrm{CW})$ and counterclockwise (CCW) components (Emery and Thomson 1997; MacKinnon et al. 2013b). For linear internal waves, a sense of CW rotation with increasing depth is consistent with downward (upward) energy (phase) propagation, while CCW rotation is consistent with upward energy propagation (Leaman and Sanford 1976; MacKinnon et al. 2013b). In addition, we will integrate over the tidal and subharmonic frequency bands to determine the amount of spectral energy in each band. We will employ the subharmonic energy ratio (SER; Chou 2013; Chou et al. 2014) criterion, based on the power spectral density (PSD), given by

$$
\text { SER }=\frac{\text { PSD at subharmonic band }}{\text { PSD at tidal band }},
$$

to quantify the fraction of energy in the subharmonic motions. In the rest of the paper, we present the results of the different analyses.

\section{Results of global energy balance analysis}

The barotropic to baroclinic conversion rates inform us about regions where internal tide energy is available to potentially contribute to mixing, the energy flux identifies wave propagation, and the divergence of the energy flux quantifies energy sources and sinks (Nash et al. 2005). For the semidiurnal tides, the map of conversion rates from our simulations (Figs. 2a,b) are comparable to previous studies (e.g., Egbert and Ray 2003; Simmons et al. 2004; Niwa and Hibiya 2011; Buijsman et al. 2016), showing concentrated activity at hotspots such as midocean ridges and shelf slopes. We find greater conversion in HYCOM25S than in HYCOM12S, especially in the Atlantic Ocean. Because the internal tides are generated by flow over topography, a higher-resolution model leads to better-resolved topographic features and hence greater conversion rates. The conversion of the diurnal tides largely lie within their turning latitudes (between latitudes $30^{\circ} \mathrm{N}$ and $30^{\circ} \mathrm{S}$ ), where theory predicts they can freely propagate (Fig. 2c). However, diurnal tides may also exist poleward of their turning latitudes as coastally trapped waves and as topographically generated waves in highly constricted tidal straits (Niwa and Hibiya 2011), as seen, for example, around the Aleutian Islands. We find here that most of the diurnal conversion is concentrated around the western side of the Pacific and the Indian Oceans, consistent with Niwa and Hibiya (2011).

The globally integrated conversion rates in HYCOM12S and HYCOM25S are $C=0.49 \mathrm{TW}$ and $0.61 \mathrm{TW}$, respectively (Figs. 2e,f). The amount of conversion from HYCOM12S is comparable to the $0.53 \mathrm{TW}$ obtained by Buijsman et al. (2016) using a HYCOM simulation forced by the eight major diurnal and semidiurnal tides. The parameterized wave drag used here dissipates about $D_{w l}=0.20 \mathrm{TW}$ of baroclinic energy in HYCOM12S and about $0.28 \mathrm{TW}$ in HYCOM25S. We note that the wave drag also dissipates about $0.71 \mathrm{TW}$ of barotropic energy in HYCOM12S and $0.61 \mathrm{TW}$ in HYCOM25S (not shown). The dissipation due to the low-mode tides, $D_{l}$, is about the same as the conversion rate since the global integral of the flux divergence is close to zero [see Eq. (1)]. As explained in Buijsman et al. (2016), the baroclinic dissipation by bottom friction can be negative, as we see in Figs. 2e and $2 \mathrm{f}$, as a result of the linear separation employed in the calculations. The linear separation method is used because of its simplicity; a nonlinear approach yields additional cross terms. Figure A1 in Buijsman et al. (2016) compares the two methods, showing that the nonlinear approach results in dissipative bottom friction.

The globally integrated barotropic-to-baroclinic diurnal conversion is $0.08 \mathrm{TW}$, about $16 \%$ of the HYCOM12S amount. Niwa and Hibiya (2011) obtain approximately $0.12(0.13) \mathrm{TW}$ from the sum of $\mathrm{K}_{1}$ and $\mathrm{O}_{1}$ in a simulation run at $1 / 10^{\circ}\left(1 / 15^{\circ}\right)$ horizontal resolution. Most of the diurnal energy conversion takes place in the western Pacific and Indian Oceans; very 

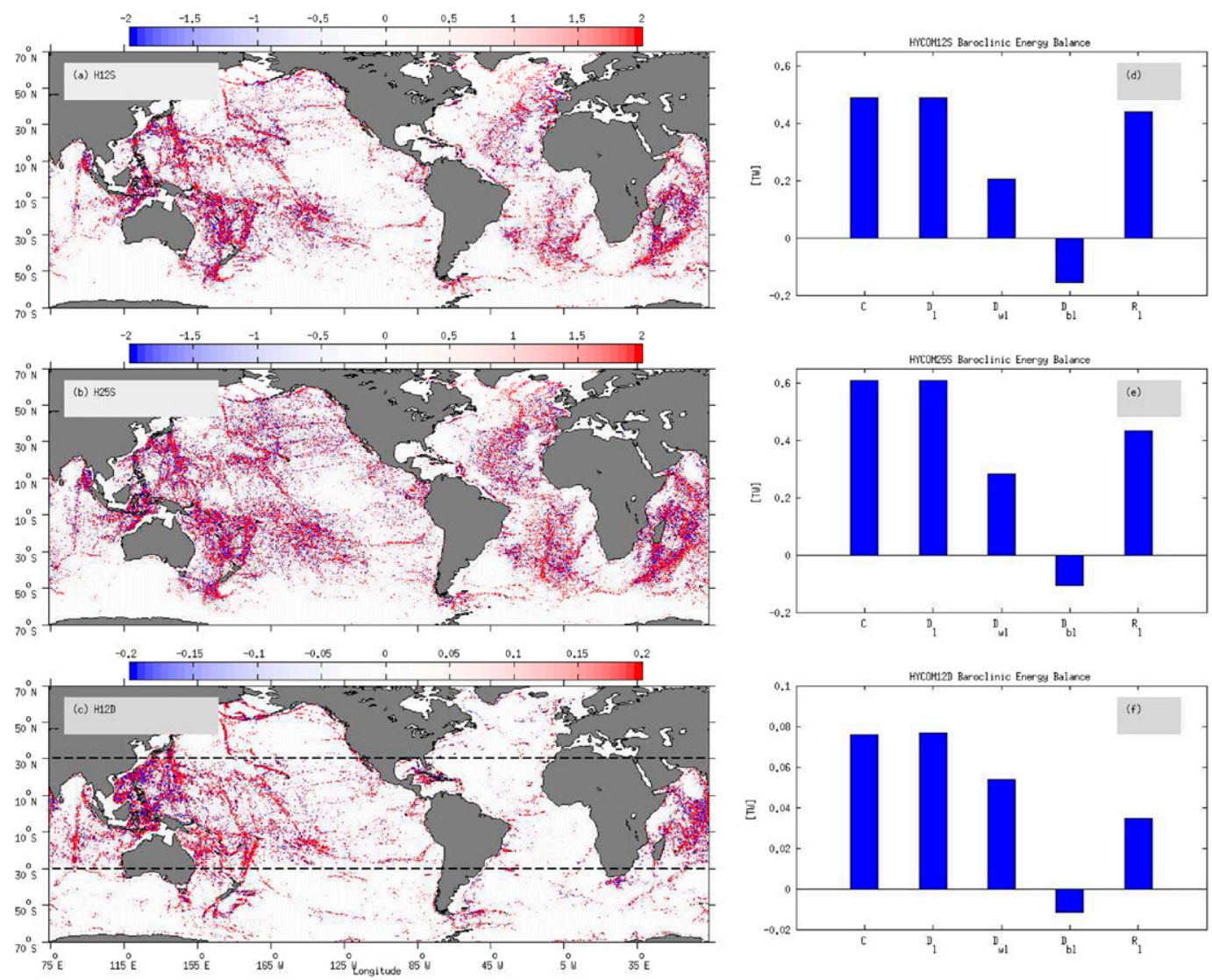

FIG. 2. (left) Barotropic to baroclinic energy conversion $\left(\mathrm{mW} \mathrm{m}^{-2}\right.$ ) for (a) HYCOM12S (b) HYCOM25S, and (c) HYCOM12D. (right) Baroclinic energy balance analysis for corresponding resolutions in the left panels, with barotropic to baroclinic conversion $C$, low-mode dissipation $D_{l}$, topographic wave drag dissipation $D_{w l}$, dissipation due to bottom friction $D_{b l}$, and residual term $R_{l}$.

little takes place in the Atlantic. This is consistent with previous studies (e.g., Egbert and Ray 2003; Niwa and Hibiya 2011). The topographic wave drag in our diurnal simulation dissipates approximately $0.05 \mathrm{TW}$ of energy, about $60 \%$ of the diurnal conversion. This diurnal wave drag to conversion ratio is larger than the $40 \%$ seen in the semidiurnal tides. The larger diurnal ratio is likely because the wave drag in HYCOM is tuned for the semidiurnal tides and therefore may "overdamp" the diurnal energy.

In both the semidiurnal and diurnal tides, a substantial fraction of the baroclinic energy budget, about $R_{l}=$ $0.4 \mathrm{TW}$ for the semidiurnal tides and $0.03 \mathrm{TW}$ for the diurnal tides, is unaccounted for. This residual energy dissipation may be attributed to viscous and numerical dissipation in the model (Buijsman et al. 2016), as well as loss to subharmonic signals via wave-wave interactions such as the PSI mechanism. A previous study by Müller et al. (2015), which employed HYCOM simulations with the same horizontal resolutions used here, showed that energy is transferred out of the low-mode inertial and semidiurnal internal waves into a broad continuum of higher-frequency and higher-wavenumber internal waves. Here, one of our goals is to estimate the amount of energy transferred from the low-mode internal tides to PSI subharmonic signals and to determine whether this amount could account for a significant fraction of the residual term in the baroclinic tidal energy budget.

\section{Subharmonic tidal signals}

\section{a. Example calculations}

Figure 3a shows an example time series of the bandpassed northward velocity component of the subharmonic NIWs, in the upper $2000 \mathrm{~m}$, at location MP3 $\left(28.93^{\circ} \mathrm{N}, 163.44^{\circ} \mathrm{W}\right.$; MacKinnon et al. 2013b) from HYCOM12S. After bandpassing for the subharmonic signal, we then employ rotary spectral analysis to decompose it into CW (Fig. 3b) and CCW (Fig. 3c) motions. At this location we see that there is subharmonic energy in both 

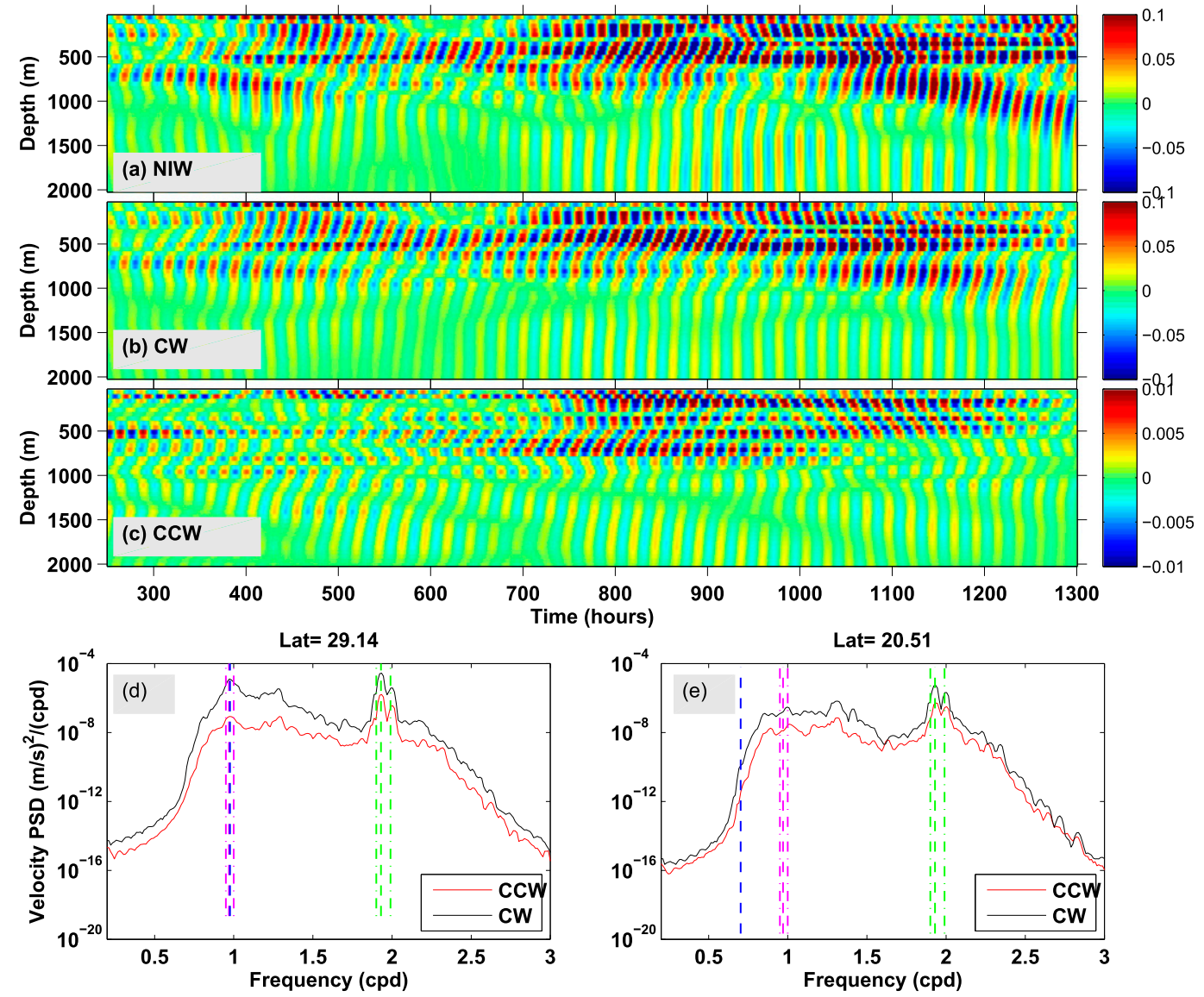

FIG. 3. (a)-(c) Time series of northward velocity $\left(\mathrm{m} \mathrm{s}^{-1}\right)$ from HYCOM12S near location MP3 $\left(29.1^{\circ} \mathrm{N}, 163.5^{\circ} \mathrm{W}\right)$ around Hawaii showing the bandpassed signals at the (a) subharmonic near-inertial frequency band (NIW), and the component of the subharmonic signal rotating (b) CW and (c) CCW. (d) The PSD of the signals in (b) and (c), and (e) the PSD at location $\left(20.5^{\circ} \mathrm{N}, 163.5^{\circ} \mathrm{W}\right)$. The green and magenta dashed lines indicate the frequencies of the semidiurnal tidal constituents and their subharmonics, respectively, and the blue dashed line show the local inertial frequency.

directions of motion but with greater energy in motions with $\mathrm{CW}$ rotation. This is shown quantitatively in Fig. 3d, where the PSD in the motions with CW rotation is greater than the PSD for motions with $\mathrm{CCW}$ rotation. Moreover, the energy in the $\mathrm{CW}$ subharmonic band is about the same as the energy in $\mathrm{CW}$ motions in the tidal band. This is likely due to the presence of local nearinertial oscillations (NIOs), which have the same frequency as the subharmonic waves at this latitude and could increase the energy in the subharmonic band. The numerical simulations of Hazewinkel and Winters (2011) show that the PSI mechanism appears to generate NIWs in the upper ocean with subsequent propagation into the ocean interior, similar to the locally generated NIOs, which are constrained to propagate into the ocean interior. This implies that it is impossible to use only PSD to separate the near-inertial waves from these two mechanisms at the critical latitude of the semidiurnal tides. That is the reason for further employing bispectral analysis (see section 7 and appendix), to help identify interactions that are largely caused by the PSI mechanism. Thus, the subharmonic signals considered in this section likely contain both the wind-generated NIW as well as those from PSI. The PSD at a location equatorward of the CL, where the tidal subharmonic signals and the local near-inertial oscillations are easily separated, is depicted in Fig. 3e; showing that the strengths of the upward and downward subharmonic motions are much closer to each other.

\section{b. Evidence for PSI}

We present below a few example calculations showing that the characteristics of the subharmonic signals in 

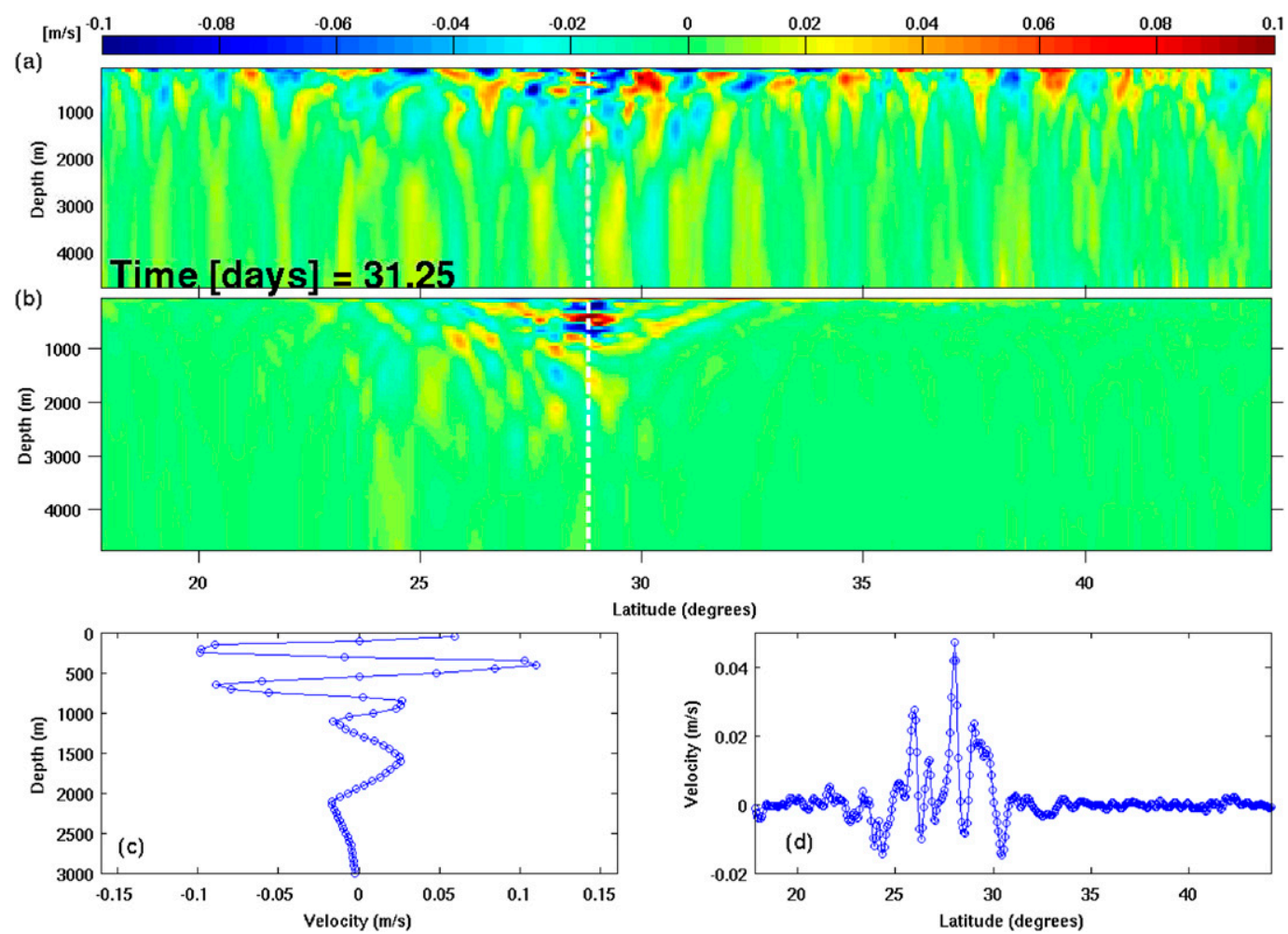

FIG. 4. (a) Snapshot of semidiurnal tidal signal of meridional baroclinic velocity from HYCOM12S close to location MP3 $\left(29.1^{\circ} \mathrm{N}, 163.5^{\circ} \mathrm{W}\right)$ around Hawaii. (b) As in (a), but after bandpassing around the subharmonic frequency band. Observe the high vertical wavenumber structure in the upper ocean around the CL. (c) A vertical profile through the $\mathrm{M}_{2}$ critical latitude $\left(\sim 28.8^{\circ}\right)$ from (b), showing the vertical scale of the subharmonic signal. (d) A horizontal slice of (b) through 1000-m depth, showing the horizontal scale of the subharmonic signal in the critical latitude band $\left(25^{\circ}-30^{\circ} \mathrm{N}\right)$.

certain locations are consistent with those of inertial waves generated via the PSI mechanism. Figures $4 \mathrm{a}$ and $4 \mathrm{~b}$ are a snapshot of baroclinic meridional velocity through location MP3 around Hawaii $\left(28.8^{\circ} \mathrm{N}, 163.5^{\circ} \mathrm{W}\right)$ depicting both the tidal and subharmonic motions from HYCOM12S. We see a higher vertical wavenumber signal in the upper ocean around the $\mathrm{M}_{2} \mathrm{CL}$. Outside the $\mathrm{CL}$ region the subharmonic signals are weaker but they have comparable strength to the tide around the CL. The NIWs from PSI have vanishing vertical velocities at the CL (e.g., MacKinnon et al. 2013b), and so we expect a concentration of subharmonic energy around the CL. Though the wind-generated NIWs have the same frequency as the subharmonic signals at the $\mathrm{CL}$, they are free to propagate away and so are not expected to have energy concentrated around the CL. We also note that the high vertical wavenumber disturbance seen in Fig. $4 \mathrm{~b}$ is similar to disturbances found by Simmons (2008) in a global model run without atmospheric wind forcing (see his Fig. 2).

We briefly discuss below the vertical and horizontal scales of the subharmonic signals around the CL. The study by Hazewinkel and Winters (2011) suggests that
PSI transfers energy to the smallest vertical scales allowed by the formulation of the problem. The model used here has thinner isopycnal layers in the upper ocean, and somewhat thicker layers with depth. The average thickness of isopycnal layers between 100 and $1000 \mathrm{~m}$ is about $100 \mathrm{~m}$. Thus, because PSI is large in the upper ocean where we have thinner layers, we expect to have our best chance of resolving the daughter waves there. On average, the subharmonic signals in Fig. 4c have vertical wavelength $\lambda_{m}$ of about $300 \mathrm{~m}$ in the upper $\sim 2000 \mathrm{~m}$ of the ocean. The vertical scales are smaller in the upper ocean and increase in scale with depth. This is consistent with observations in MacKinnon et al. (2013b) around the same location, where they estimate a vertical wavelength of about $200 \mathrm{~m}$ (in the $\sim 400-750-\mathrm{m}$ depth range) with larger scales at depth. The vertical scales are also comparable to those in observations by Alford et al. (2007), though the magnitudes of the velocities are much smaller in our model. Other observational studies around Hawaii find the subharmonic waves from PSI to have vertical scales between 50 and $150 \mathrm{~m}$ (Sun and Pinkel 2013). 

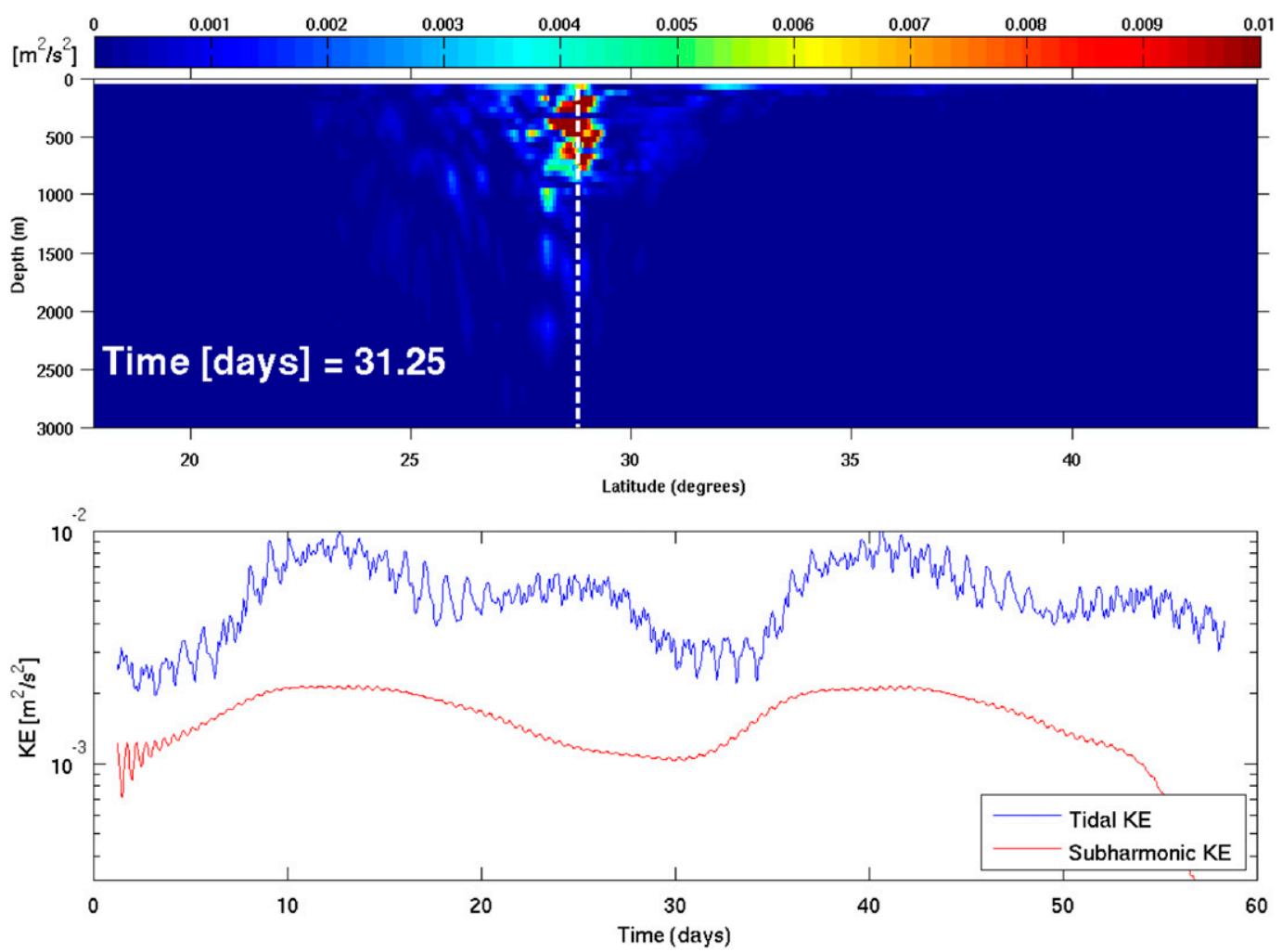

FIG. 5. (a) Snapshot of the subharmonic baroclinic kinetic energy density $\left(u^{2}+v^{2}\right)$ from the vertical section in Fig. 4b, computed from HYCOM12S. (b) Temporal variation of vertically averaged subharmonic kinetic energy density from the vertical section in (a).

Horizontal-wavenumber spectra computed from the tidal and subharmonic bands at different depths in the upper ocean show a range of lateral scales (not shown). The tides peak at wavelengths of around $90-150 \mathrm{~km}$, and the subharmonics have wavelengths in the $60-100-\mathrm{km}$ range. For example, we estimate a horizontal wavelength $\lambda_{k}$ of about $100 \mathrm{~km}$ around the CL band at a depth of about $1000 \mathrm{~m}$. This horizontal wavelength is comparable to the lateral scales of near-inertial waves reported from observations (Alford et al. 2017). Figure 4d shows that the horizontal scale of the subharmonic signal is not uniform across the CL. As a check, we can separately compute the frequency of the waves $\omega$ associated with $\lambda_{m}=300 \mathrm{~m}$ and $\lambda_{k}=100 \mathrm{~km}$ from the dispersion relationship for internal gravity waves:

$$
\omega^{2}=\frac{m^{2} f^{2}+k^{2} N^{2}}{m^{2}+k^{2}}=\frac{f^{2}+(k / m)^{2} N^{2}}{1+(k / m)^{2}}
$$

where $m=2 \pi / \lambda_{m}$ is the vertical wavenumber, $k=2 \pi / \lambda_{k}$ is the horizontal wavenumber, $f$ is the Coriolis frequency, and $N$ is the buoyancy frequency. Because $(k / m) \approx 3 \times 10^{-3}$ and $N \approx 3 \times 10^{-5} \mathrm{~s}^{-1}$, we see from Eq. (12) that $\omega \approx f$, indicating that the waves are near-inertial as expected. The subharmonic baroclinic $\mathrm{KE}$ density from the vertical section in Fig. $4 \mathrm{~b}$ is displayed in Fig. 5a, further emphasizing the upper-ocean intensification of subharmonic KE density around the CL. We find from Fig. 5b that the vertically averaged subharmonic KE densities roughly follow the spring-neap cycle of the tidal KE, signifying that they are likely generated via the PSI mechanism (MacKinnon and Winters 2005; Alford et al. 2007; Hazewinkel and Winters 2011). The bispectrum and bicoherence calculations (briefly explained in section 7 and the appendix) at this location are displayed (in Fig. A1), showing significant bicoherence at several vertical levels.

\section{Global PSD calculations}

At each grid location, we integrate the PSD over the tidal and subharmonic bands for both the $\mathrm{CW}$ and $\mathrm{CCW}$ components. We emphasize that the PSD calculations from the subharmonic band alone are insufficient to distinguish PSI subharmonics from other oceanic motions of similar frequency in a model that includes atmospheric forcing in addition to tidal forcing. The calculations here present only an initial map of the 


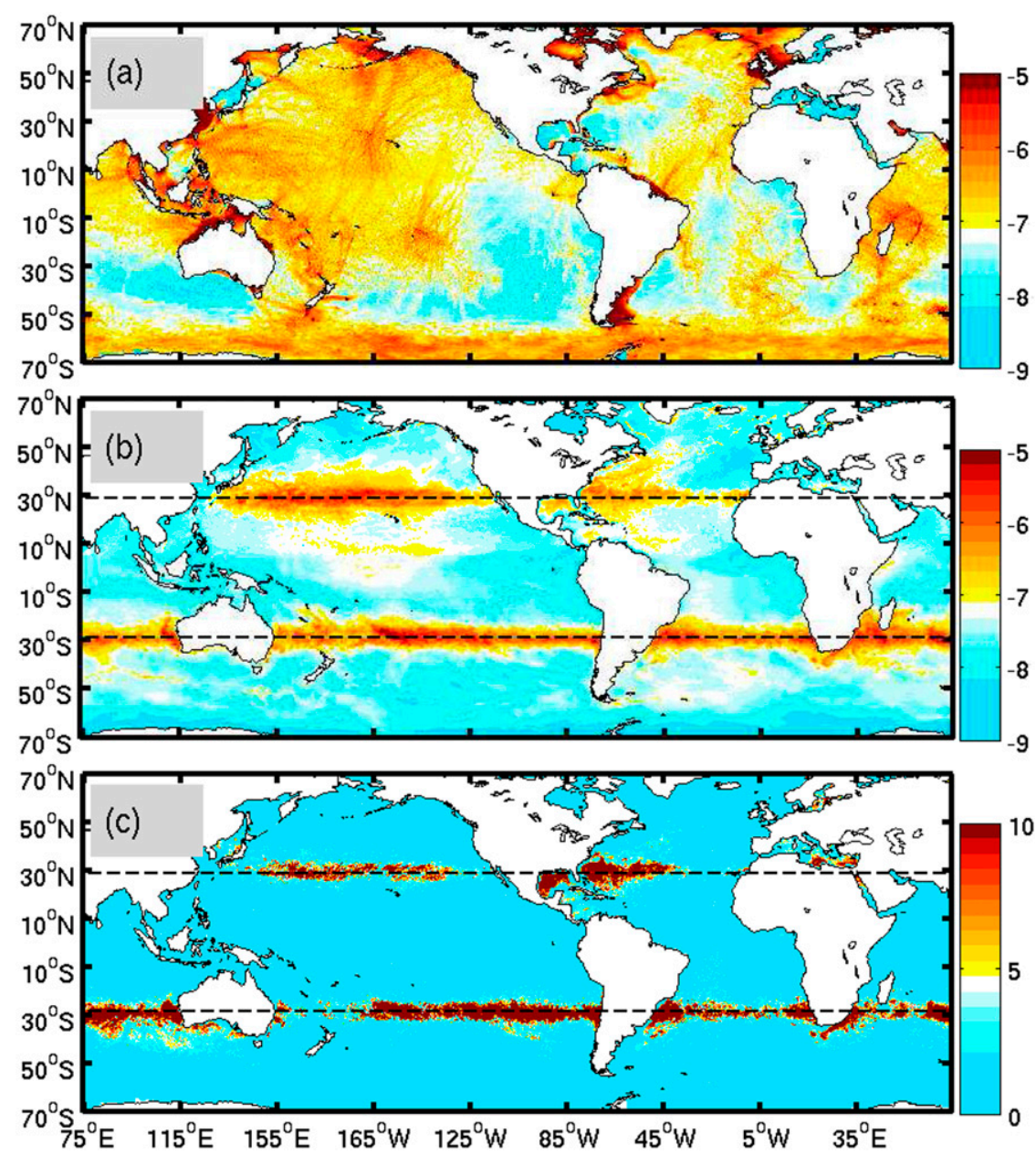

FIG. 6. Global distribution of bandpassed and vertically integrated PSD from HYCOM12S for the (a) semidiurnal band, (b) subharmonic band, and (c) SER. Note that the maximum SER in (c) is about 400 .

distribution of variance in the total subharmonic band. Figure $6 \mathrm{a}$ is the band-integrated and vertically integrated PSD from the HYCOM12S tidal band. For brevity, we display only the sum of the $\mathrm{CW}$ and $\mathrm{CCW}$ components of the integrated PSD. The map reveals the hotspots of tidal activity similar to the map of conversion (see Fig. 2a). Figure 6b is similar to Fig. 6a but for subharmonic signals with half the frequency of the tide. We see a distribution of large subharmonic PSD around the CL, as in the results of Simmons (2008, the bottom panel of his Fig. 3). However, in our results there is a greater latitudinal spread around the $\mathrm{CL}$ as well as a continuous longitudinal distribution of these subharmonic signals, in contrast to the patchy signals along the CL observed in Simmons (2008). These differences in our results are probably caused by the interactions of the tides with other motions and by the presence of atmospherically forced motions such as mesoscale eddies and wind-generated near-inertial waves. The presence of atmospheric forcing in our high-resolution model ensures a vigorous mesoscale eddy field, comparable to observations and yields large variabilities in the internal tides in both the model and observations (Ansong et al. 2017). Figure 6c shows the SER [Eq. (11)], which has a maximum value of about 400 . Most large values of SER are concentrated around the CL. We employ the SER later to filter out less energetic subharmonic signals outside the critical latitude regions, especially in our bicoherence calculations. The vertically averaged PSDs from HYCOM25S show larger kinetic energy variances in both the tidal and subharmonic bands than in HYCOM12S. Moreover, the SER from HYCOM25S (not shown) is also concentrated around the CL but appears patchier than the HYCOM12S results in Fig. 6c.

The distribution of vertically integrated PSD from the diurnal frequency band is shown in Fig. 7a. We find that 

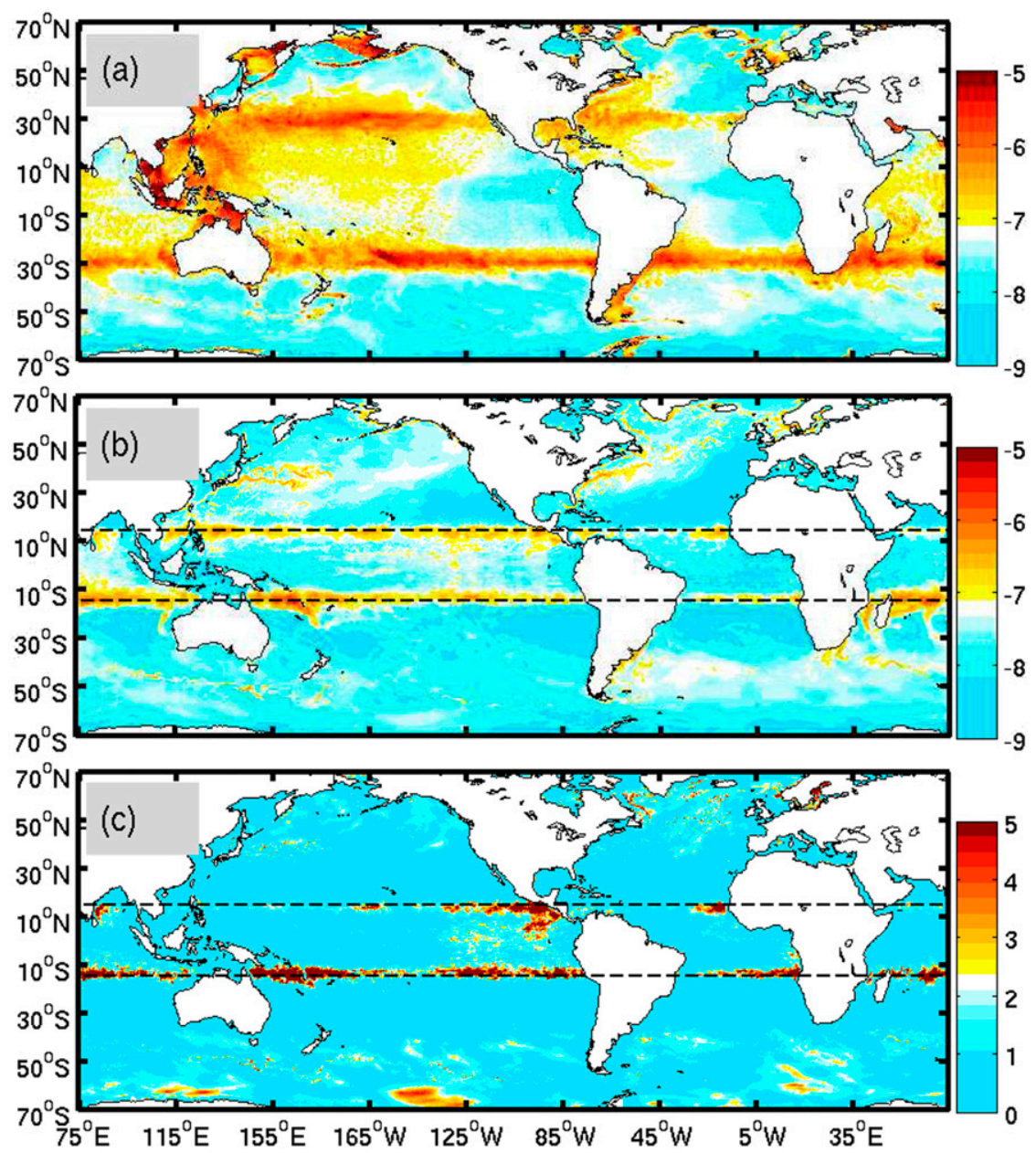

FIG. 7. As in Fig. 6, but for HYCOM12D.

most of the diurnal kinetic energy variance occurs equatorward of their turning latitudes $\left( \pm 30^{\circ}\right)$, as expected from theory, as well as in certain regions such as the Aleutian Arc and the Sea of Okhotsk, likely caused by topographically trapped waves (Egbert and Ray 2003; Niwa and Hibiya 2011) as mentioned earlier. The subharmonic signals from the diurnal band are much weaker than their semidiurnal counterparts (Fig. 7b), but they also have larger variances along their CL. The map of SER in this case (Fig. 7c) reveals patchy locations of high subharmonic variance along the CL. We also find relatively high variance occurring poleward of the $\mathrm{CL}$, in both the diurnal and semidiurnal PSD. This is likely caused, in part, by leakage of mesoscale motions into the subharmonic band.

\section{Results of bispectral analyses}

When different spectral components interact nonlinearly, the resulting signal contains frequencies of the primary waves as well as daughter waves with sum and difference frequencies of the primary waves. To quantify nonlinear interactions, one must resort to higherorder spectral analysis techniques (Nikias and Petropulu 1993). For signals arising from wave-wave interactions, the cumulant bicoherence spectrum can be used to measure the extent of the joint dependence of the spectral components (Kim and Powers 1979). Unlike the power spectrum, the bicoherence spectrum may be used to separate nonlinearly coupled waves (which exhibit phase coherence) from spontaneously excited independent waves, without reference to the waves' amplitudes (Kim and Powers 1979). These authors also show that the squared bicoherence can be used to measure the fraction of power in a given spectral band due to the quadratic coupling. We have given a brief summary of this statistical technique and the related concept of bispectrum in the appendix.

Other previous investigations of PSI in the ocean have employed bispectral analysis (Hasselmann et al. 1963; Furue 2003; Furuichi et al. 2005; Carter and Gregg 2006; 
Frajka-Williams et al. 2006; MacKinnon et al. 2013b; Sun 2010; Sun and Pinkel 2012, 2013; Chou et al. 2014; Xie et al. 2016). Because bispectral analysis was developed for stationary and random processes, its application to nearly deterministic signals like the tides is challenging (Chou 2013). Another difficulty is the possibility of obtaining significant bicoherence levels from synthetic signals that are not interacting nonlinearly with each other (Chou et al. 2014). The work of Sun (2010) and MacKinnon et al. (2013b) provides useful information on the application of bispectral analysis to internal tides, as employed in this study.

In the following, we use the SER, as well as bispectrum and bicoherence calculated at each model layer, to determine the global patterns of bicoherence from our simulations. Using the $\mathrm{CW}$ and $\mathrm{CCW}$ components of the subharmonic complex velocity time series, we calculate the bicoherence at each grid point using the approach of Kim and Powers (1979). The calculation yields a value between 0 and 1 at each grid location, with 1 indicating high bicoherence (implying high phase correlation between the tides and the subharmonic signals). We use significance levels for the bicoherence developed by Elgar and Guza (1988). In our case values of 0.35 (for HYCOM12S and HYCOM12D) and 0.5 (for HYCOM25S) indicate bicoherence at the $95 \%$ significant levels respectively (see the appendix). We plot bicoherences at locations where both the peak bispectrum and significant bicoherence occur within the subharmonic frequency band.

\section{a. Geographical distribution of bicoherence}

Figures $8 \mathrm{a}$ and $8 \mathrm{~b}$ show the results of the vertically averaged bicoherence calculations for HYCOM12S, while Figs. 8c and 8d show the same results for HYCOM25S. It is likely that in some locations, especially poleward of the $\mathrm{CL}$, we will find subharmonic signals with significant bicoherence alongside small energy levels, as demonstrated by the SER calculations in section 6. We use the SER here to filter out weak subharmonic signals that have significant bicoherence values. For example, in Fig. 8a, locations with SER $<1$ are not plotted for clarity. Figure 8 a displays a concentration of significant bicoherence values around the CL. Neglecting signals with SER $<2$ further narrows the distribution of the energetic signals with significant bicoherence to the CL (Fig. 8b). The observational study of Xie et al. (2016) suggests that near-inertial waves induced by PSI can be transported poleward beyond their CL by background geostrophic flow. Similarly, Richet et al. (2017) find two weak dissipation peaks of internal tides due to PSI at latitudes $\sim 25^{\circ}$ and $\sim 35^{\circ}$, from idealized numerical studies, and attributed this to the presence of a mean flow in their domain. Thus, the effects of the background flow might partially explain the presence of significant bicoherence values poleward of the CL. The bispectral results for HYCOM25S are displayed in Figs. 8c and 8d and illustrate a similar distribution of significant bicoherence as in HYCOM12S. The bicoherence takes values between 0 and 1 . Here, we find that most of the significant bicoherence values in HYCOM25S generally lie closer to 1 (with a global average of about 0.73 ), whereas the values in HYCOM12S lie in the lower bound of the bicoherence range (with a global average of about 0.43 ). This implies that the phase correlations between the tides and the subharmonic signals in HYCOM25S are generally stronger than in HYCOM12S. Thus, higher-resolution simulations are likely to facilitate greater wave-wave interactions between the tides and the subharmonics. The bicoherence values in the diurnal case are generally much smaller than in HYCOM12S, as displayed in Fig. 9, and appear to be contaminated by the mesoscale motions, which are present in the bandpassed signals.

\section{b. Vertical distribution of bicoherence}

Figure 10a shows a zonally averaged meridional section of bicoherence driven by semidiurnal tides in the Northern Hemisphere for HYCOM12S. Significant bicoherence values occur throughout the water column around the CL with a peak just equatorward of the CL (Figs. 10a,b). We observe that even though PSI may occur anywhere equatorward of the CL, its intensity quickly falls off about $5^{\circ}$ from the CL. This result is consistent with the two-dimensional idealized results of Furuichi et al. (2005, see their Fig. 5). Figures 10c and 10d show that the significant bicoherence values occurring away from the CL region have smaller energy (based on the SER criterion). Figures 10c and 10d also show that the interactions that are both energetic and significant occur in the upper ocean and are concentrated around the CL. For instance, the significant bicoherence values occurring in the deep ocean in Fig. 10a have smaller averaged SER values than those in the upper ocean. Therefore, the prominence of the deep ocean after the SER criteria has been applied is less, as seen in Fig. 10d. As discussed in previous studies (MacKinnon and Winters 2005; Young et al. 2008; Hazewinkel and Winters 2011), the subharmonic motions draw their energy from horizontal gradients of the internal tides [see Eq. (9) and following discussion]. Thus, in a model with surface-intensified stratification and consequent surface-intensified internal tides, PSI is expected to be concentrated in the upper ocean as we find here. Because the rates of energy transfer in PSI are proportional to the energy in the waves, transfer terms such as $u \partial u / \partial x$ are amplified in highly stratified locations 


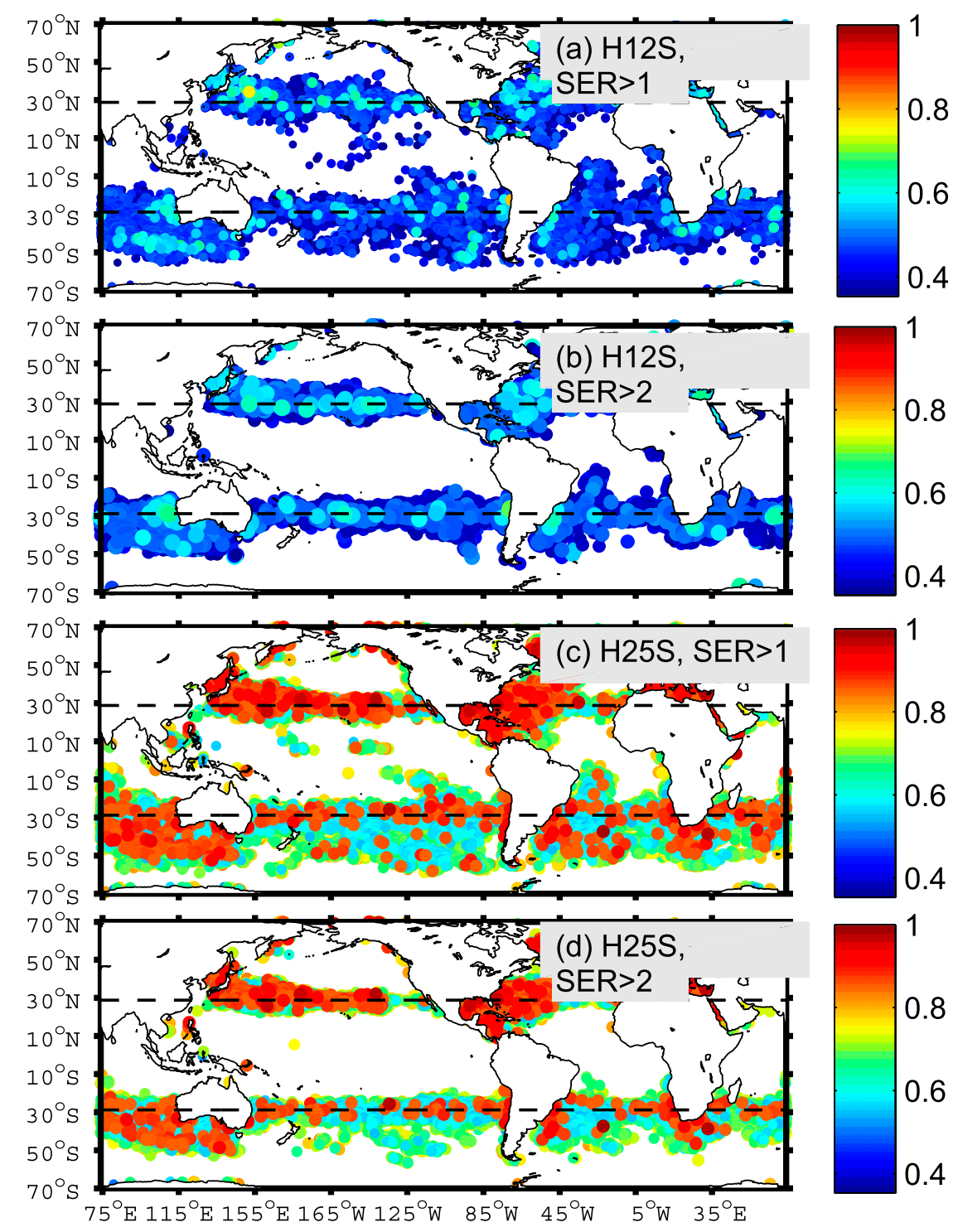

FIG. 8. Global distribution of vertically averaged bicoherence for (a) HYCOM12S (SER > 1), (b) HYCOM12S (SER > 2), (c) HYCOM25S (SER > 1), and (d) HYCOM25S (SER > 2). The plots display significant bicoherence values at the $95 \%$ significance level $\left(b_{95 \%}=0.35\right.$ for HYCOM12S and 0.50 for HYCOM25S), and every sixth grid point is plotted.

such as the thermocline (Frajka-Williams et al. 2006; MacKinnon et al. 2013a). As further presented in Carter and Gregg (2006), the ratio of nonlinear to linear terms in the equations of motion, such as $u \partial u / \partial x /(\partial u / \partial t)$, scales as the square root of the buoyancy frequency, $\sqrt{N(z)}$, such that the nonlinear interactions could be expected to be larger in regions of strong stratification.

The center panels in Fig. 10 display the vertical distribution of zonally averaged bicoherence for HYCOM25S. The pattern of bicoherence is similar to that of HYCOM12S but generally shows higher bicoherence values, as mentioned earlier. In addition, we see that the region around the CL with significant bicoherence values is broader in HYCOM25S (Fig. 10e) than in HYCOM12S and displays two smaller peaks in the vertically averaged plot (Fig. 10f). In contrast to HYCOM12S, we find significant bicoherence values deeper in the ocean in HYCOM25S (Fig. 10h). Figure 10 (right panels) are the counterparts of Fig. 10 (left panels) but for HYCOM12D. Similar to the distribution in HYCOM12S, we find a concentration of significant bicoherence values centered around the diurnal CL over all depths. In this case, we also see significant bicoherence values poleward of the CL (Figs. 10i,j) as also reported by Xie et al. (2016). 

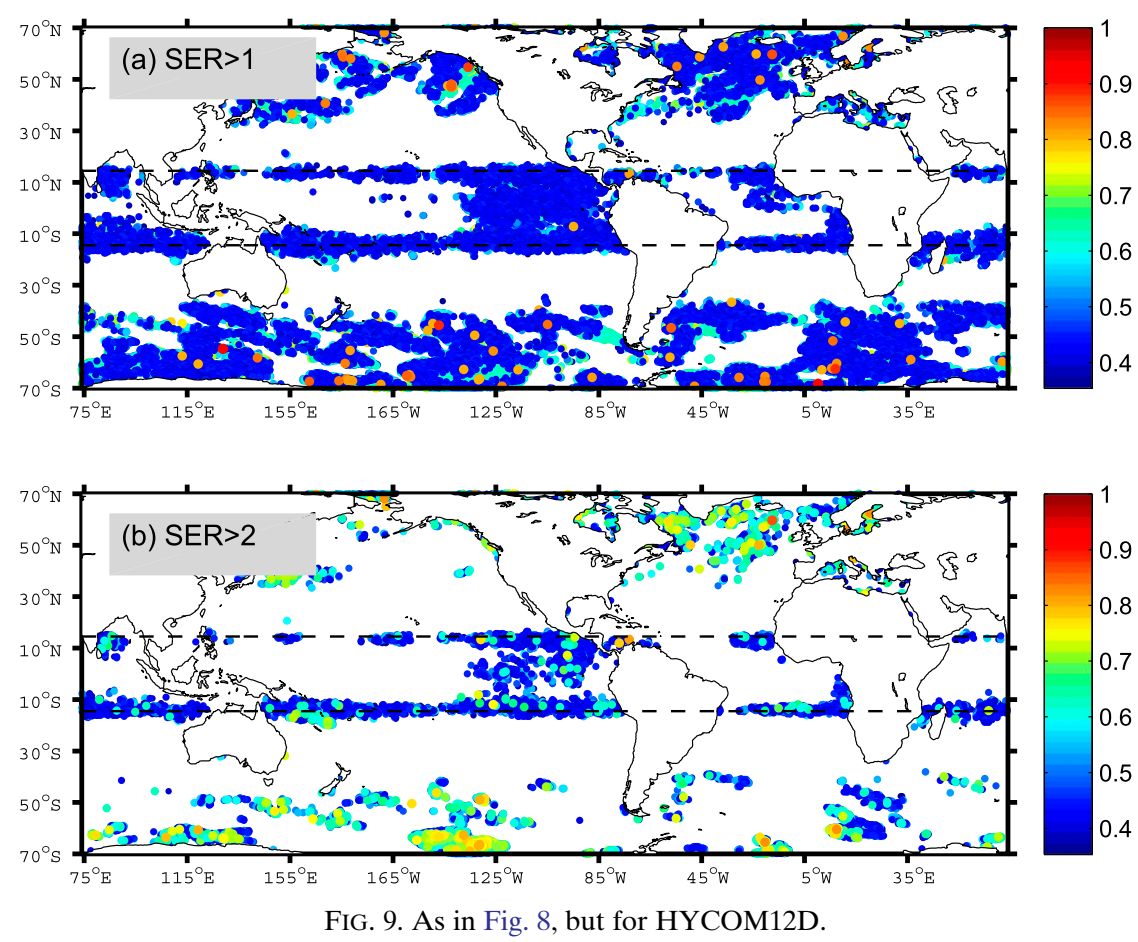

However, as shown in Figs. 10k and 101, these poleward bicoherence values are weaker than those equatorward of the CL.

\section{Energy transfer rates}

We next present estimates of energy transfer rates computed using Eq. (9). We find that the term $-\mathbf{u}_{1}^{*} \cdot\left(\mathbf{u}_{3} \cdot \nabla \mathbf{u}_{2}\right)$ is negligible everywhere compared to $T=-\mathbf{u}_{1}^{*} \cdot\left(\mathbf{u}_{2} \cdot \nabla \mathbf{u}_{3}\right)$ as a primary driver of PSI, as suggested by previous studies (not shown for brevity; see section 3 ). We thus compute the energy transfer rates using the latter term in which $\mathbf{u}_{1}$ and $\mathbf{u}_{2}$ are the CW and CCW components of the bandpassed subharmonic velocities and $\mathbf{u}_{3}$ is the tidal velocity. Figure 11a displays a meridional section of energy transfer rates in the North Pacific region from HYCOM12S. We see relatively large energy transfers in the upper $100 \mathrm{~m}$ of the ocean, the result of mixed layer processes. Below $100 \mathrm{~m}$, we find patchy locations of positive energy transfers (transfers from the tidal band to subharmonic signals) as well as negative/reverse transfers (transfers from the subharmonics to the tidal band), consistent with previously reported observational results around Hawaii (MacKinnon et al. 2013b; Sun and Pinkel 2013). The energy transfers in this case are largely located in the upper $1000 \mathrm{~m}$ of the ocean. A vertical profile of transfer rates around location MP3 is displayed in Fig. 11b (blue curve) showing positive transfers over most of the profile below 200-m depth. A few degrees to the east of
MP3, we find relatively large transfers between $300-$ and 550-m depth (Fig. 11b, magenta curve). We note that the energy transfer rates $\left(\sim 10^{-10} \mathrm{~W} \mathrm{~kg}^{-1}\right)$ from both profiles are about an order of magnitude smaller than in observations around the same location (MacKinnon et al. 2013b, their Fig. 11). A horizontal slice of transfer rates around MP3 at about 400-m depth is displayed in Fig. 11c. We see about the same amounts of positive and negative transfers around MP3 with a maximum transfer rate of about $1.1 \times$ $10^{-9} \mathrm{~W} \mathrm{~kg}^{-1}$ at $\left(29.2^{\circ} \mathrm{N}, 197.68^{\circ} \mathrm{E}\right)$. The bispectral calculations near MP3 are given in the appendix (Fig. A1), and show significant bicoherence values in the 300-1000-m depth range. Figures $11 \mathrm{~d}$ and $11 \mathrm{e}$ are the counterparts of Figs. 11a and 11b but for HCYOM25S. In this case, we find more locations with relatively large transfer rates in the vertical section than in HYCOM12S. Unlike HYCOM12S, the energy transfers also extend much deeper into the water column ( $\sim 2500-\mathrm{m}$ depth, not shown). The vertical profile of energy transfer near MP3 (Fig. 11e, blue curve) shows largely negative transfers in HYCOM25S, unlike what we find in HYCOM12S (Fig. 11b, blue curve). Thus, the dominant sign of energy transfer with depth appears to be influenced by local conditions and model resolution. Energy transfers at the location just east of MP3 (Fig. 11e, magenta curve) appear to have a similar vertical structure as in HYCOM12S, with largely positive energy transfers with depth.

Figures $12 \mathrm{a}$ and $12 \mathrm{~b}$ show vertically integrated global energy transfer rates from the semidiurnal band. 

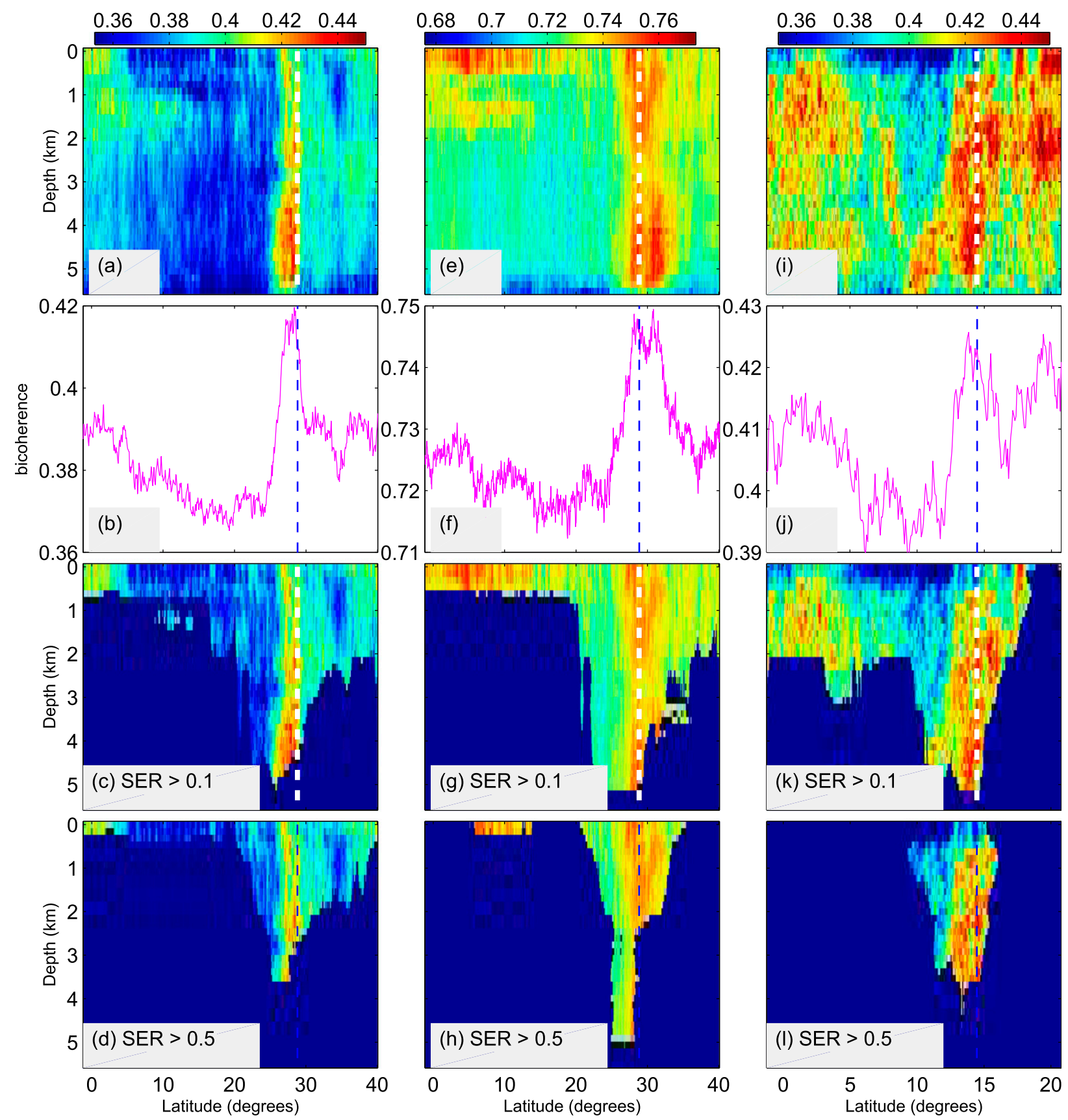

FIG. 10. (a) Variation in depth and latitude of zonally averaged bicoherence for HYCOM12S. (b) Vertically averaged bicoherence values in (a), showing a drop-off in intensity of bicoherence away from the critical latitude. The dashed vertical lines indicate the position of the $\mathrm{M}_{2}$ critical latitude. (c),(d) As in (a), but with zonally averaged SER $>0.1$ and SER $>0.5$, respectively. (e)-(h) As in (a)-(d), but for HYCOM25S. (i)-(1) As in (a)-(d), but for HYCOM12D.

The figures display a concentration of relatively large transfer rates around the $\mathrm{CL}$ after employing the SER criterion. Comparing Fig. $6 \mathrm{~b}$ to the more rigorous calculation of energy transfers in Fig. 12a, we see that some of the signals appearing in Fig. $6 \mathrm{~b}$ along the critical latitude (e.g., in the southeast Pacific region) vanish. This indicates that those subharmonic signals may have come from other motions rather than from PSI. Similar to the semidiurnal energy transfers, the diurnal transfer rates are both positive and negative, with a net positive transfer. In addition, the diurnal transfer rates are generally much smaller than the semidiurnal transfers 

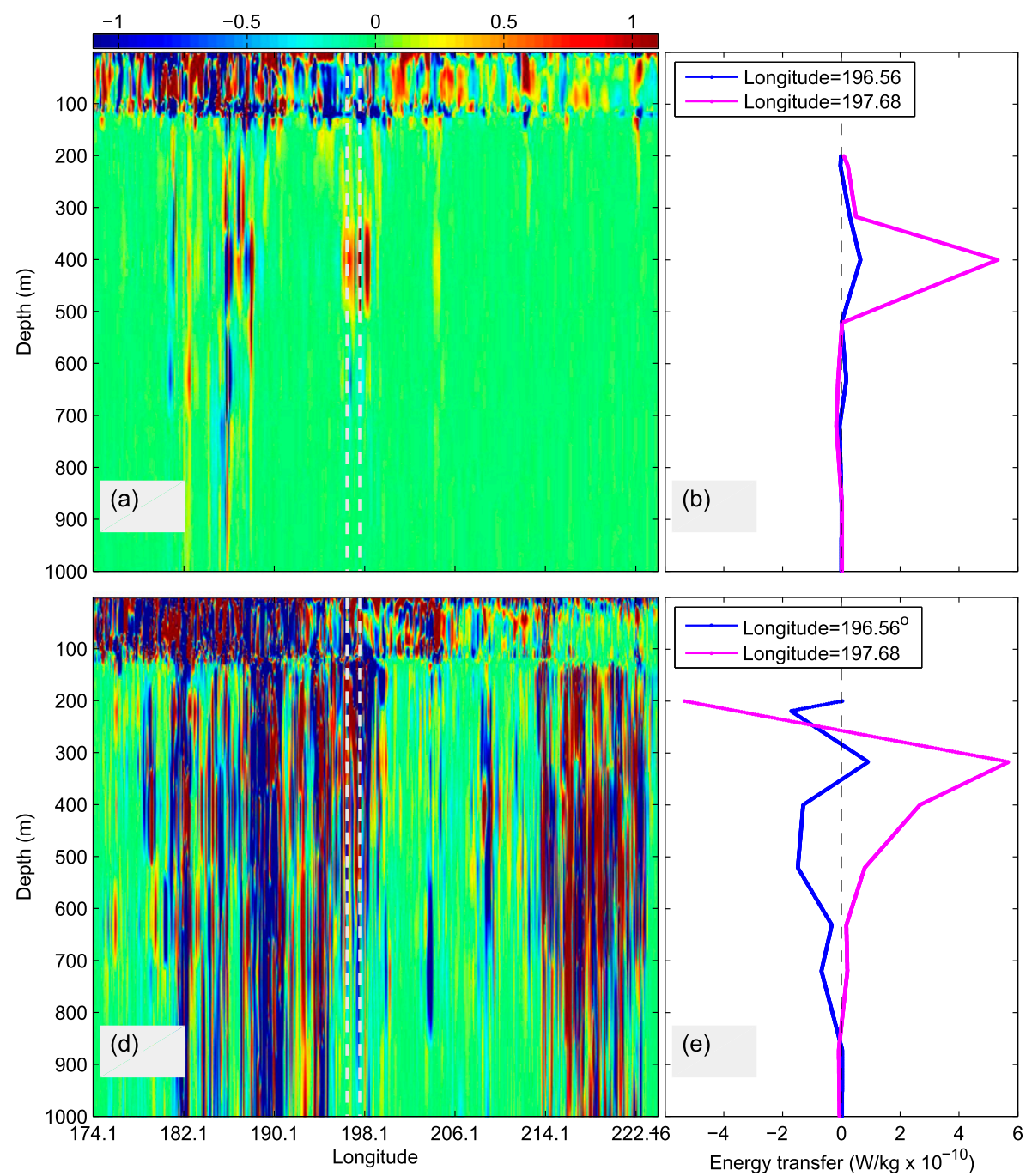

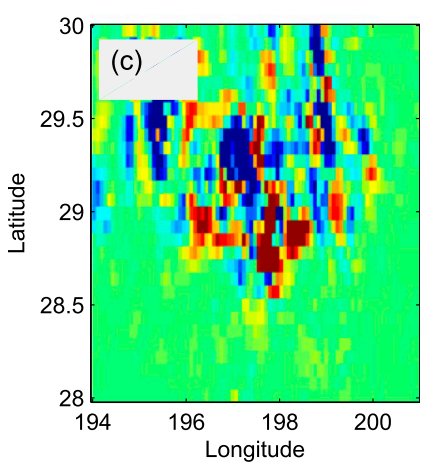

FIG. 11. Energy transfer rates from HYCOM12S (a) at a zonal section along latitude $29.14^{\circ} \mathrm{E}\left(\times 10^{-10} \mathrm{~W} \mathrm{~kg}^{-1}\right)$, (b) in a vertical profile near location MP3 (blue curve) and just to the east of MP3 (magenta curve), and (c) at a depth of about $400 \mathrm{~m}$ near MP3. (d),(e) As in (a) and (b), but for HYCOM25S. The white dashed lines in (a) and (d) show the positions of profiles given by the blue and magenta curves in (b) and (e).

(Fig. 12c; note the different color scale used for clarity). As discussed in section 4, this is likely due to the fact that the diurnal internal tides are generally weaker than the semidiurnals, due in part to the effect of the topographic wave drag scheme that dissipates a comparatively larger fraction of diurnal energy. We see relatively large diurnal energy transfers in patchy locations in the east equatorial Pacific region and the southwestern Pacific region (to the northeast of Australia) around the diurnal CL. These locations also show high diurnal subharmonic variance as seen in Figs. $7 \mathrm{~b}$ and $7 \mathrm{c}$. We also find that the diurnal transfer rates appear contaminated by the mesoscale motions, especially around the Gulf Stream, Kuroshio, and Antarctic Circumpolar Current
(Fig. 12c), likely because diurnal tides are less energetic than semidiurnal tides.

Figure 12d displays the zonally averaged semidiurnal energy transfer rates for the positive and negative components. We see that the transfers are almost equally partitioned between the positive and negative transfers with a net positive transfer over the globe. The globally integrated positive and negative energy transfers are $+0.197 \mathrm{TW}$ and $-0.196 \mathrm{TW}$, respectively, for HYCOM12S, resulting in a net amount of $\sim 0.001 \mathrm{TW}$. This amount is about $0.5 \%$ of the $0.40 \mathrm{TW}$ residual energy transfer needed to close the HYCOM12S baroclinic energy budget. In the case of HYCOM25S, the globally integrated amount of energy transfer is much 

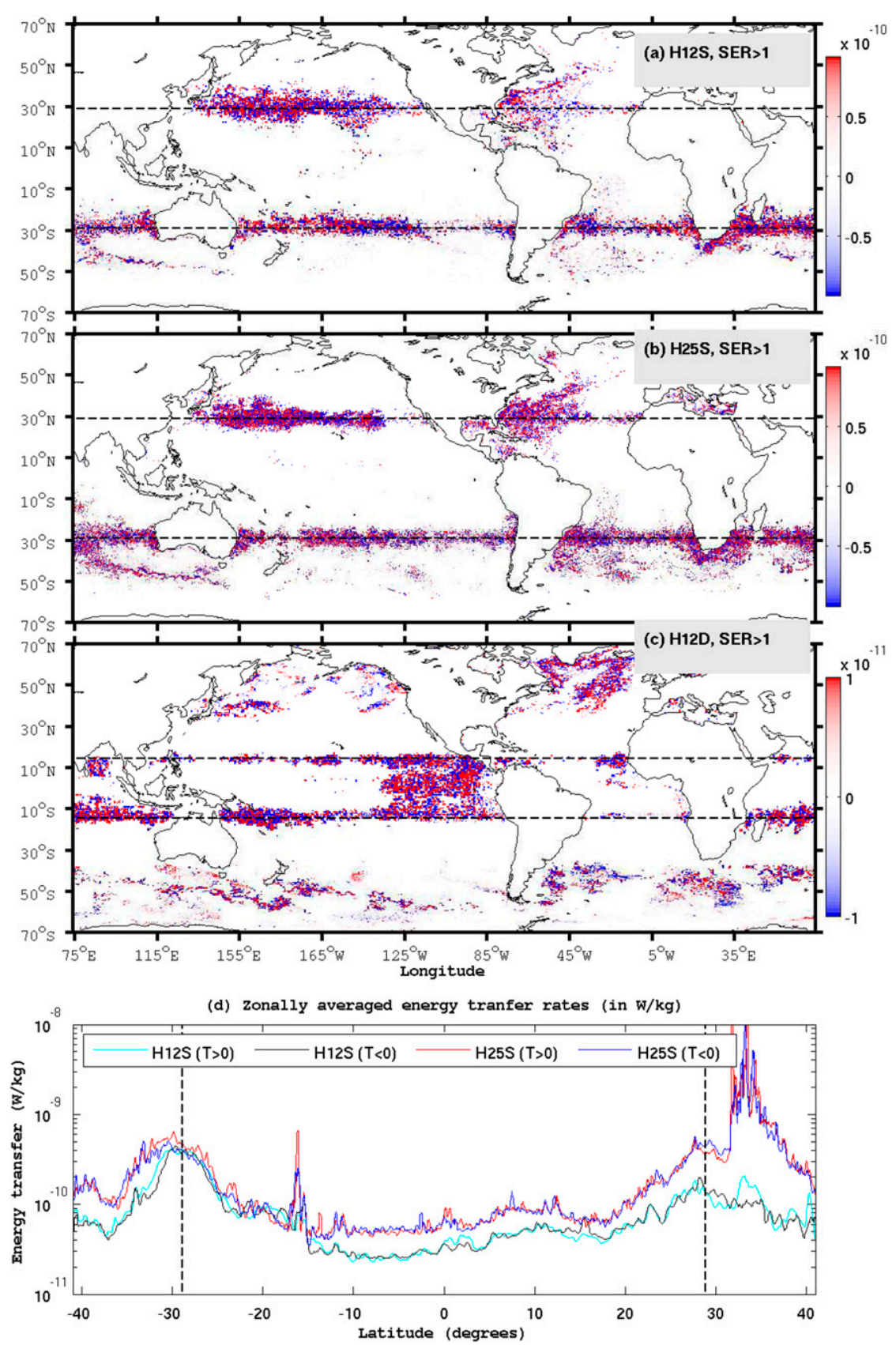

FIG. 12. Vertically integrated global distribution of energy transfer rates $\left(\mathrm{W} \mathrm{kg}^{-1}\right)$ between low-mode tides and subharmonic signals for (a) HYCOM12S, (b) HYCOM25S, and (c) HYCOM12D. (d) Zonally averaged energy transfer rates from HYCOM12S (H12S) and HYCOM25S (H25S), showing that the positive and negative transfers have about the same magnitude, leading to a negligible net energy transfer. " $T$ " is used to denote the sign of energy transfer.

larger, with values of $+0.48 \mathrm{TW}$ and $-0.44 \mathrm{TW}$, giving a net amount of $0.04 \mathrm{TW}$. This amount is about $10 \%$ of the residual energy transfer. Thus, increasing the horizontal model resolution increases the amount of energy transfer by about an order of magnitude. The globally integrated positive and negative amounts of energy for HYCOM12D are $+0.086 \mathrm{TW}$ and $-0.074 \mathrm{TW}$, respectively, giving a net value of $0.012 \mathrm{TW}$. The net amount of energy transfer in this case is about $4.0 \%$ of the residual $(\sim 0.03 \mathrm{TW})$. 
We point out that the residual energy flux going into PSI is a small difference of large numbers and changes with model resolution, making it difficult to estimate the uncertainty in the energy transfer values. For example, the globally integrated positive and negative transfers from HYCOM25S are each about $0.5 \mathrm{TW}$, which we assume to be a measure of the standard deviation. To measure the standard error, we need an estimate of the number of degrees of freedom (DOF). This is a difficult quantity to measure, so we give a crude estimate here. Let us take $\mathrm{DOF}=\mathrm{NT} \times \mathrm{NW} \times \mathrm{NV}$, where $\mathrm{NT}$ is the number of degrees of freedom in time, NW is the number of waves involved in a global estimate, and NV is the number of degrees of freedom in the vertical direction. Because the internal tides are dominated by mode- 1 waves, let us take NV $=1$. We take NT to be the number of $\mathrm{M}_{2}$ periods in the HYCOM25S record, namely, $30 \times 24 / 12.42 \approx 58$. For NW, we count the number of internal tide beams emanating from source regions in HYCOM to be about 90 (e.g., from Fig. 2a in Ansong et al. 2017). This yields DOF $=5220$. So a rough estimate of the standard error is $0.5 / \sqrt{5220}=0.007 \mathrm{TW}$, which is about $18 \%$ of the mean value of $0.04 \mathrm{TW}$. Given the uncertainties in the estimate of DOF and the fact that we are dealing with residuals of differences of large numbers, we cannot state with confidence that the sign of the residuals is positive in the simulations. The large increase in both positive and negative transfers with increases in resolution further increases our uncertainties and leaves open the possibility that still higher-resolution simulations will find somewhat different answers, thus opening the door for future studies.

\section{Summary and discussion}

Diurnal and semidiurnal internal tide energy analyses are performed using three simulations of the HYCOM global ocean circulation model at $1 / 12.5^{\circ}$ and $1 / 25^{\circ}$ horizontal resolutions. HYCOM12S and HYCOM12D are used to denote the HYCOM simulations forced by the semidiurnal and diurnal tides, respectively, at $1 / 12.5^{\circ}$ resolution, and $\mathrm{HYCOM} 25 \mathrm{~S}$ represents the $1 / 25^{\circ}$ semidiurnal simulation. For the semidiurnal tides, the parameterized topographic wave drag in HYCOM12S (HYCOM25S) dissipates about $0.20(0.28)$ TW of baroclinic energy, representing about $41 \%(46 \%)$ of the globally integrated barotropic-to-baroclinic conversion $[\sim 0.49(0.61) \mathrm{TW}]$. The semidiurnal amount of conversion is comparable to previous computations (Egbert and Ray 2003; Simmons et al. 2004; Niwa and Hibiya 2011; Buijsman et al. 2016). Compared to the semidiurnal tides, the globally integrated conversion in the diurnal tides is much smaller $(0.08 \mathrm{TW})$; about $60 \%$ of the generated internal tides is dissipated by the wave drag. A significant fraction of baroclinic energy dissipation of the semidiurnal tides $(\sim 0.40 \mathrm{TW})$ and the diurnal tides $(\sim 0.03 \mathrm{TW})$ is not accounted for in our baroclinic energy balance. This amount of residual energy dissipation is attributed to viscous and numerical dissipation in the model as well as loss to subharmonic signals via wavewave interactions. A previous study using HYCOM simulations, with the same horizontal resolutions employed here, showed that energy is transferred out of the low-mode inertial and semidiurnal internal waves into a broad continuum of higher-frequency and higherwavenumber internal waves (Müller et al. 2015). Thus, one of the goals of the present study is to determine whether a significant percentage of the residual energy dissipation in the model is due to energy transfer to subharmonic waves via the PSI mechanism.

In this paper, we used power spectral density and energy transfer calculations, as well as bispectral analysis, to provide evidence for the occurrence of PSI in HYCOM and to map out the geographic distribution of the hypothesized PSI. In contrast to Simmons (2008), who focused on $\mathrm{M}_{2}$-only simulations, here we employed two simulations forced by three semidiurnal $\left(\mathrm{N}_{2}, \mathrm{M}_{2}, \mathrm{~S}_{2}\right)$ and one simulation forced by three diurnal $\left(\mathrm{Q}_{1}, \mathrm{O}_{1}, \mathrm{~K}_{1}\right)$ tidal constituents. All simulations also included atmospheric forcing, thereby ensuring a vigorous mesoscale eddy field. Energy transfer computations using equations similar to previous studies (e.g., MacKinnon et al. 2013b; Sun and Pinkel 2013) show that the largest energy transfers are near the CLs, with most large transfers confined to the upper ocean. However, we find that the energy transfers are almost equally partitioned between positive transfers (from low-mode tides to subharmonic signals) and negative/reverse transfers (Fig. 12d), in contrast to previous idealized simulations (MacKinnon and Winters 2005). We compute a net positive energy transfer (energy loss from the tide due to PSI) in all simulations with varying amounts. The net global energy loss is $\sim 0.001 \mathrm{TW}$ in HYCOM12S, which is $\sim 0.5 \%$ of the residual energy needed to close the HYCOM12S baroclinic energy budget. The net global amount of energy transfer in HYCOM25S is relatively larger, about $0.04 \mathrm{TW}$, representing approximately $10 \%$ of the residual transfer. This shows that the rate of energy transfer is sensitive to the model resolution, with the finer resolution facilitating greater wave-wave interactions and larger energy transfer rates. For the diurnal tides, we estimate a net global amount of energy transfer of 0.012 TW in HYCOM12 simulations, representing about $4.0 \%$ of the residual transfer.

In addition to energy transfer calculations, we performed bispectral analysis to provide evidence for, and 
map the geographic distribution of, PSI in the model. Direct application of the bicoherence method of Kim and Powers (1979) shows evidence of PSI around the CL together with statistically significant bicoherence values appearing in high-latitude regions. The geographic distribution of bicoherence is more localized to the CL upon employing the criterion that the SER [Eq. (11)] is at least 1.

We have shown that, over all depths, the zonally averaged bicoherence from both the diurnal and semidiurnal bands is concentrated around the critical latitudes, and that the intensity of the zonally averaged bicoherence significantly decreases about $5^{\circ}$ away from the CL. This is consistent with the previous idealized results of Furuichi et al. (2005), who also found that the intensity of PSI in the Pacific quickly drops over a distance of about $3^{\circ}$ from the CL. The most energetic subharmonic signals with significant bicoherence values also show high intensity in the upper ocean. This is consistent with the idealized simulations of Hazewinkel and Winters (2011), who attributed the surface intensification of PSI to the surface-intensified structure of the buoyancy frequency. A reviewer pointed out that an alternative hypothesis for the observation that PSI daughter waves are enhanced at the critical latitudes is that they could be waves that are generated by other processes, but with an amplitude that is enhanced at the turning latitude. This alternative hypothesis cannot be nullified by our model results. However, we think the hypothesis is unlikely for several of the reasons presented in the paper. For instance, if the waves were generated by other random processes in the model, they would not likely lead to an enhancement of waves with significant bicoherence values and relatively higher energy transfers along the critical latitudes. Second, the theory of PSI energy transfers shows that near the critical latitude the primary driver of PSI is the term $T_{1}=-\mathbf{u}_{1}^{*} \cdot\left(\mathbf{u}_{2} \cdot \nabla \mathbf{u}_{3}\right)$ rather than the term $T_{2}=-\mathbf{u}_{1}^{*} \cdot\left(\mathbf{u}_{3} \cdot \nabla \mathbf{u}_{2}\right)$. Our results are consistent with theory, because we find that indeed $T_{2}$ is negligible everywhere on the globe whereas the term $T_{1}$ produces transfers that are enhanced along the critical latitudes. The analysis of $T_{1}$ and $T_{2}$ also shows that the subharmonic waves seen in our results draw their energy from the horizontal gradients of the tidal velocities, as found in observations (e.g., MacKinnon et al. 2013a). A third piece of evidence is that the high vertical wavenumber disturbance seen at the critical latitude in our analyses (e.g., Fig. 4b) is qualitatively similar to that of Simmons (2008) in a model that was run without atmospheric wind forcing. Last, the estimated vertical scales of the subharmonic waves are close to those seen in observations of PSI daughter waves (e.g., Alford et al. 2007; MacKinnon et al. 2013a), and we have shown that they are indeed near inertial (see our example calculations in section 5b). A combination of all the reasons mentioned above gives us some confidence that the subharmonic waves are likely of PSI origin.

Previous studies suggest that energy transfers to the daughter waves of PSI might be faster between the subharmonics and higher-mode internal tides (mode $\geq 3$ ) than between the subharmonics and the low mode- 1 waves (MacKinnon et al. 2013b). The HYCOM model presented here well resolves the mode- 1 and mode- 2 waves but barely resolves modes 3 and higher. This might be a possible reason for our low estimate of the net global energy flux to the subharmonics. Thus, a suggestion (O. Sun 2013, personal communication) for a future study is to separate out the higher-mode tides and to use them to compute bicoherences and energy transfer rates. Another future endeavor is to use harmonic analysis to separate out individual tidal constituents, in contrast to bandpassed signals, to investigate PSI.

We emphasize that the idealized simulations in Hazewinkel and Winters (2011) imply that results in any model, including ours, are dependent upon the model resolution and viscosities. They find a $15 \%$ tidal energy loss to PSI using a vertical eddy viscosity of $5 \times$ $10^{-5} \mathrm{~m}^{2} \mathrm{~s}^{-1}$ and a $25 \%$ reduction by prescribing a zero viscosity. We find here that most of the significant bicoherence values in HYCOM25S generally lie closer to the upper value of 1 , whereas the significant values in HYCOM12S lie in the lower range. This implies that the phase correlations between the tides and the subharmonic signals in HYCOM25S are generally higher than in HYCOM12S. Thus, simulations with horizontal resolutions even higher than $1 / 25^{\circ}$ are likely to facilitate greater wave-wave interactions between the tides and the subharmonics. The simulations reported here are too expensive to do sensitivity studies on different viscosities and vertical resolutions. It is expected that the estimates of PSI energy transfer given here will be improved upon in the future by running higher-resolution simulations and by storing and processing longerduration records from the model, so that different motions can be more accurately separated.

There are still open questions about PSI. One question is about the long-term behavior of PSI. For instance, how does the rate of energy transfer and geographical distribution of PSI change seasonally? This question could not be addressed here because of the short length of time series used. Studies have begun to explore the time-varying mixing signals of PSI origin (Qiu et al. 2012). In addition, there are still challenges in isolating the subharmonic waves of PSI in the real ocean (and in a complex model like ours), where many frequencies are present. The recent study by Richet et al. (2017) suggests 
that the frequencies of the PSI subharmonic waves likely depend upon both the frequency and horizontal wavenumber of the primary tide, as well as on mean currents, as a result of Doppler effects. Following previous work, this study assumed that the frequencies of the PSI daughter waves are about half the tidal frequencies, and it would be interesting to investigate the effects of modifying the subharmonic frequencies, as suggested by Richet et al. (2017), in a global model. We hope to address these and other interesting scientific questions in future studies.

Acknowledgments. This research represents a contribution to the Climate Process Team (CPT) project "Collaborative Research: Representing Internal-Wave Driven Mixing in Global Ocean Models," which focuses on improving estimates of mixing due to internal waves in the ocean. The project is funded by the National Science Foundation and is led by Jennifer MacKinnon of the Scripps Institution of Oceanography. We are grateful to Jennifer MacKinnon, Oliver Sun, Eric D'ASaro, Eric Kunze, and Sherry Chou for extremely helpful discussions. We are also grateful to Tim Duda for suggesting that we look at energy levels as well as significance of the bispectra. Finally, we would like to acknowledge very helpful and thorough comments from two anonymous reviewers of this manuscript and an earlier version of this manuscript, and from a third anonymous reviewer of the earlier version. J.K.A. and B.K.A. acknowledge funding from the University of Michigan Associate Professor Support Fund, supported by the Margaret and Herman Sokol Faculty Awards. J.K.A. and B.K.A. gratefully acknowledge support from National Science Foundation CPT Grant OCE-0968783 and Office of Naval Research Grant N00014-11-1-0487. B.K.A. and P.G.T. acknowledge support from a University of Texas Jackson School of Geosciences Development grant, Naval Research Laboratory (NRL) contract N000173-06-2-C003, Office of Naval Research Grants N00014-09-1-1003 and N00014-11-10487, and National Science Foundation Grant OCE0924481. H.L.S. was supported by NSF (CPT) Grant OCE-0968838 and ONR Grant N00014-09-1-0399. M.H.A. was supported by Grant OCE-0968131. J.G.R., J.F.S., and A.J.W. were supported by the projects "Eddy resolving global ocean prediction including tides" and "Ageostrophic vorticity dynamics" sponsored by the Office of Naval Research under Program Element $0602435 \mathrm{~N}$. This work was supported in part by a grant of computer time from the DOD High Performance Computing Modernization Program at the Navy DSRC. This is NRL contribution NRL/JA/ 7320-13-1693.

\section{APPENDIX}

\section{Bispectral Analysis}

Given a zero mean signal $x(t)$ with its complex Fourier transform $X(\omega)$, the bispectrum is defined as (Kim and Powers 1979)

$$
B\left(\omega_{1}, \omega_{2}\right)=E\left[X\left(\omega_{1}\right) X\left(\omega_{2}\right) X^{*}\left(\omega_{3}\right)\right],
$$

where $t$ is time, $\omega$ is frequency, $E[\cdot]$ is the expectation operator, and $X^{*}$ is the complex conjugate of $X$. The implication of this definition is that the bispectrum is identically zero unless 1 ) the frequency components $\omega_{1}, \omega_{2}$ and $\omega_{3}=\omega_{1}+\omega_{2}$ are present in a given signal and 2) phase coherence (or phase consistency) is also present among the three components. A quantitative measure of the bispectrum is the bicoherence defined by Kim and Powers (1979) as

$$
b^{2}\left(\omega_{1}, \omega_{2}\right)=\frac{\left|B\left(\omega_{1}, \omega_{2}\right)\right|^{2}}{E\left[\left|X\left(\omega_{1}\right) X\left(\omega_{2}\right)\right|^{2}\right] E\left[\left|X\left(\omega_{3}\right)\right|^{2}\right]},
$$

with $0 \leq b\left(\omega_{1}, \omega_{2}\right) \leq 1$. Alternate normalizations of the bispectrum have also been presented in previous studies (Elgar and Guza 1988; Hinich and Wolinsky 2005; Sun and Pinkel 2012).

In practical applications, the bicoherence is estimated as

$$
\tilde{b}=\frac{\tilde{B}}{\tilde{E}\left[\left|X\left(\omega_{1}\right) X\left(\omega_{2}\right)\right|\right] \tilde{E}\left[\left|X\left(\omega_{3}\right)\right|\right]},
$$

where

$$
\begin{aligned}
\tilde{B} & =\left|\frac{1}{M} \sum_{i}^{M} X\left(\omega_{1}\right)^{(i)} X\left(\omega_{2}\right)^{(i)} X\left(\omega_{3}\right)^{*}(i)\right| \\
\tilde{E}\left[\left|X\left(\omega_{1}\right) X\left(\omega_{2}\right)\right|\right] & =\left[\frac{1}{M} \sum_{i}^{M}\left|X\left(\omega_{1}\right)^{(i)} X\left(\omega_{2}\right)^{(i)}\right|^{2}\right]^{1 / 2}, \\
\tilde{E}\left[\left|X\left(\omega_{3}\right)\right|\right] & =\left[\frac{1}{M} \sum_{i}^{M}\left|X\left(\omega_{3}\right)^{(i)}\right|^{2}\right]^{1 / 2}
\end{aligned}
$$

Here, $M$ is the set of data records each of length, say, $N$. We divided our time series into $50 \%$ overlapping windows, each of length $N=256 \mathrm{~h}$, and applied a Hamming window to each record as in previous studies (Nikias and Petropulu 1993; Kim and Powers 1979).

In practice, it is likely that a finite length time series, even with truly independent components, will have nonzero bispectrum. For these reasons, Elgar and Guza (1988) have established significance levels of zero bicoherence 
(a) IBispectrum I

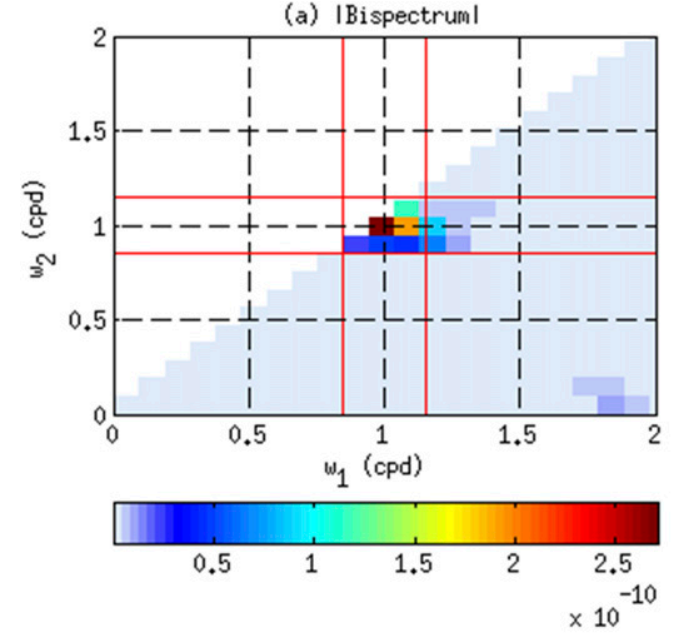

(b) Bicoherence
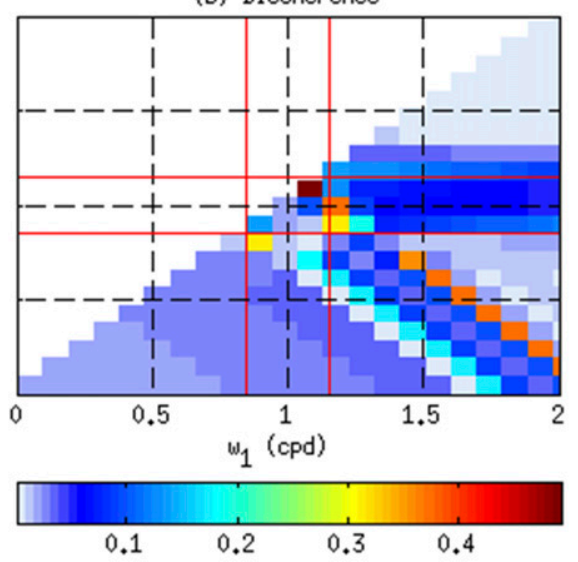

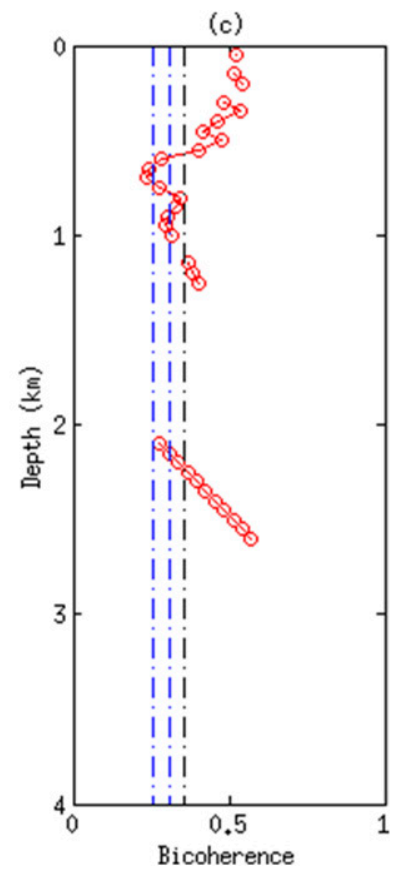

FIG. A1. Example bispectral calculation from HYCOM12S at location $\left(29.14^{\circ} \mathrm{N}, 163.5^{\circ} \mathrm{W}\right)$, depicting the (a) bispectrum and (b) bicoherence at about 500-m depth, and (c) significant bicoherence values at each vertical level. The vertical dashed lines in (c) show the value at the $80 \%, 90 \%$, and $95 \%$ significance levels.

to help determine if data are statistically consistent with a linear, random phase process. They find significance levels of $b$ at $95 \% \equiv \sqrt{6 / n_{d}}$ (denoted by $b_{95}$ ) and $99 \% \equiv \sqrt{9 / n_{d}}$, where $n_{d}$ is the number of degrees of freedom. Determining the number of degrees of freedom in the case of internal waves is a problematic issue (Carter and Gregg 2006; Sun 2010; MacKinnon et al. 2013b; Sun and Pinkel 2012; Chou 2013). Following MacKinnon et al. (2013b), we estimate $n_{d}=2 \times(60 / 2.5)=$ 48 for the 60-day span of our HYCOM12S and HYCOM12D time series, and $n_{d}=2 \times(30 / 2.5)=24$ for HYCOM25S. The value 2.5 days is approximately the number of days over which tidal signals significantly change phase and/or amplitude (MacKinnon et al. $2013 b)$. We note that the value 2.5 days is somewhat subjective and is only based on the observational study by MacKinnon et al. (2013b). This means that, at each vertical level, 95\% significance levels result in bicoherence values of 0.35 and 0.50 for HYCOM12 and HYCOM25S, respectively. Note that the estimates above do not account for additional degrees of freedom resulting from vertical averaging.

An example calculation illustrating the bispectral method above is shown in Fig. A1 for a time series taken near location MP3 around Hawaii $\left(29.14^{\circ} \mathrm{N}, 163.5^{\circ} \mathrm{W}\right)$. The peak values of both the bispectrum and bicoherence, at about $500 \mathrm{~m}$ depth, lie within the subharmonic frequency band (red lines in Figs. A1a,b). The peak bicoherence is not exactly at the location of the peak bispectrum because of the normalization factor [the denominator in Eq. (A1)]. For this reason, the bispectrum and the bicoherence need to be considered together to determine nonlinear interactions. Figure A1c depicts significant bicoherence values at each vertical level, showing two main depth ranges (300-1000 m and 2100-2600 m) where the tides and the PSI subharmonics have significant bicoherence.

\section{REFERENCES}

Alford, M. H., 2008: Observations of parametric subharmonic instability of the diurnal internal tide in the South China Sea. Geophys. Res. Lett., 35, L15602, https://doi.org/10.1029/ 2008GL034720 10.1029/2008GL034720.

—, J. MacKinnon, Z. Zhao, R. Pinkel, J. Klymak, and T. Peacock, 2007: Internal waves across the Pacific. Geophys. Res. Lett., 34, L24601, https://doi.org/10.1029/2007GL031566.

, R. Pinkel, and J. M. Klymak, 2017: Space-time scales of shear in the North Pacific. J. Phys. Oceanogr., 47, 2455-2478, https://doi.org/10.1175/JPO-D-17-0087.1.

Ansong, J. K., B. K. Arbic, M. C. Buijsman, J. G. Richman, J. F. Shriver, and A. J. Wallcraft, 2015: Indirect evidence for substantial damping of low-mode internal tides in the open ocean. J. Geophys. Res. Oceans, 120, 6057-6071, https://doi.org/ 10.1002/2015JC010998.

- and Coauthors, 2017: Semidiurnal internal tide energy fluxes and their variability in a global ocean model and moored observations. J. Geophys. Res. Oceans, 122, 1882-1900, https:// doi.org/10.1002/2016JC012184. 
Arbic, B. K., S. T. Garner, R. W. Hallberg, and H. L. Simmons, 2004: The accuracy of surface elevations in forward global barotropic and baroclinic tide models. Deep-Sea Res. II, 51, 3069-3101, https://doi.org/10.1016/j.dsr2.2004.09.014.

- A. J. Wallcraft, and E. J. Metzger, 2010: Concurrent simulation of the eddying general circulation and tides in a global ocean model. Ocean Modell., 32, 175-187, https://doi.org/ 10.1016/j.ocemod.2010.01.007.

— J. J. Richman, J. F. Shriver, P. G. Timko, E. J. Metzger, and A. J. Wallcraft, 2012: Global modeling of internal tides within an eddying ocean general circulation model. Oceanography, 25 (2), 20-29, https://doi.org/10.5670/oceanog.2012.38.

Bleck, R., 2002: An oceanic general circulation model framed in hybrid isopycnic-Cartesian coordinates. Ocean Modell., 4, 5588, https://doi.org/10.1016/S1463-5003(01)00012-9.

Bourget, B., H. Scolan, T. Dauxois, M. L. Bars, P. Odier, and S. Joubaud, 2014: Finite-size effects in parametric subharmonic instability. J. Fluid Mech., 759, 739-750, https:// doi.org/10.1017/jfm.2014.550.

Buijsman, M. C., B. K. Arbic, J. A. M. Green, R. W. Helber, J. G. Richman, J. F. Shriver, P. G. Timko, and A. J. Wallcraft, 2015: Optimizing internal wave drag in a forward barotropic model with semidiurnal tides. Ocean Modell., 85, 42-55, https://doi.org/10.1016/j.ocemod.2014.11.003.

— semidiurnal energy balance in a global ocean circulation model. J. Phys. Oceanogr., 46, 1399-1419, https://doi.org/ 10.1175/JPO-D-15-0074.1.

Carter, G. S., and M. C. Gregg, 2006: Persistent near-diurnal internal waves observed above a site of $\mathrm{M}_{2}$ barotropic-tobaroclinic conversion. J. Phys. Oceanogr., 36, 1136-1147, https://doi.org/10.1175/JPO2884.1.

Chassignet, E. P., and Coauthors, 2009: Global ocean prediction with the Hybrid Coordinate Ocean Model (HYCOM). Oceanography, 22 (2), 64-76, https://doi.org/ 10.5670/oceanog.2009.39.

Chou, S. H., 2013: An empirical investigation of energy transfer from the $\mathrm{M}_{2}$ tide to $\mathrm{M}_{2}$ subharmonic wave motions in the Kauai Channel, Hawaii. M.S. thesis, Dept. of Oceanography, University of Hawai'i at Mānoa, 86 pp., http://hdl.handle.net/ 10125/101881.

—_ D. S. Luther, M. D. Guiles, G. S. Carter, and T. Decloedt, 2014: An empirical investigation of nonlinear energy transfer from the $\mathrm{M}_{2}$ internal tide to diurnal wave motions in the Kauai Channel, Hawaii. Geophys. Res. Lett., 41, 505512, https://doi.org/10.1002/2013GL058320.

Egbert, G. D., and R. D. Ray, 2003: Semi-diurnal and diurnal tidal dissipation from TOPEX/Poseidon altimetry. Geophys. Res. Lett., 30, 1907, https://doi.org/10.1029/2003GL017676.

Elgar, S., and R. T. Guza, 1988: Statistics of bicoherence. IEEE Trans. Acoust. Speech Signal Process., 36, 1667-1668, https:// doi.org/10.1109/29.7555.

Emery, W. J., and R. E. Thomson, 1997: Data Analysis Methods in Physical Oceanography. Pergamon, $654 \mathrm{pp}$.

Frajka-Williams, E. E., E. L. Kunze, and J. A. MacKinnon, 2006: Bispectra of internal tides and parametric subharmonic instability. arXiv, https://arxiv.org/abs/1410.0926.

Furue, R., 2003: Energy transfer within the small-scale oceanic internal wave spectrum. J. Phys. Oceanogr., 33, 267-287, https://doi.org/ 10.1175/1520-0485(2003)033<0267:ETWTSS > 2.0.CO;2.

Furuichi, N., T. Hibiya, and Y. Niwa, 2005: Bispectral analysis of energy transfer within the two-dimensional oceanic internal wave field. J. Phys. Oceanogr., 35, 2104-2109, https://doi.org/10.1175/JPO2816.1.
Gayen, B., and S. Sarkar, 2013: Degradation of an internal wave beam by parametric subharmonic instability in an upper ocean pycnocline. J. Geophys. Res. Oceans, 118, 4689-4698, https:// doi.org/10.1002/jgrc.20321.

Gerkema, T., C. Staquet, and P. Bouruet-Aubertot, 2006: Decay of semi-diurnal internal-tide beams due to subharmonic resonance. Geophys. Res. Lett., 33, L08604, https://doi.org/10.1029/ 2005 GL025105.

Halliwell, G. R., 2004: Evaluation of vertical coordinate and vertical mixing algorithms in the Hybrid-Coordinate Ocean Model (HYCOM). Ocean Modell., 7, 285-322, https://doi.org/ 10.1016/j.ocemod.2003.10.002.

Hasselmann, K., W. Munk, and G. MacDonald, 1963: Bispectra of ocean waves. Time Series Analysis, M. Rosenblatt, Ed., Wiley, $125-139$ pp.

Hazewinkel, J., and K. B. Winters, 2011: PSI of the internal tide on a $\beta$-plane: Flux divergence and near-inertial wave propagation. J. Phys. Oceanogr., 41, 1673-1682, https://doi.org/ 10.1175/2011JPO4605.1.

Hendershott, M. C., 1973: Inertial oscillations of tidal period. Prog. Oceanogr., 6, 1-27, https://doi.org/10.1016/0079-6611 (73)90003-7.

Hibiya, T., Y. Niwa, and K. Fujiwara, 1998: Numerical experiments of nonlinear energy transfer within the oceanic internal wave spectrum. J. Geophys. Res., 103, 18 715-18 722, https://doi.org/ 10.1029/98JC01362.

_- M. Nagasawa, and Y. Niwa, 2002: Nonlinear energy transfer within the oceanic internal wave spectrum at mid and high latitudes. J. Geophys. Res., 107, 3207, https://doi.org/10.1029/ 2001JC001210.

Hinich, M., and M. Wolinsky, 2005: Normalizing bispectra. J. Stat. Plann. Inference, 130, 405-411, https://doi.org/ 10.1016/j.jspi.2003.12.022.

Hogan, T. F., and Coauthors, 2014: The Navy Global Environmental Model. Oceanography, 27 (3), 116-125, https://doi.org/ 10.5670/oceanog.2014.73.

Jayne, S. R., and L. C. St. Laurent, 2001: Parameterizing tidal dissipation over rough topography. Geophys. Res. Lett., 28 , 811-814, https://doi.org/10.1029/2000GL012044.

Kang, D., and O. Fringer, 2012: Energetics of barotropic and baroclinic tides in the Monterey Bay area. J. Phys. Oceanogr., 42 , 272-290, https://doi.org/10.1175/JPO-D-11-039.1.

Kim, Y. C., and E. J. Powers, 1979: Digital bispectral analysis and its applications to nonlinear wave interactions. IEEE Trans. Plasma Sci., 7, 120-131, https://doi.org/10.1109/TPS.1979.4317207.

Large, W. G., J. C. McWilliams, and S. C. Doney, 1994: Oceanic vertical mixing: A review and a model with a nonlocal boundary layer parameterization. Rev. Geophys., 32, 363-403, https://doi.org/10.1029/94RG01872.

Leaman, K. D., and T. B. Sanford, 1976: Observations on the vertical polarization and energy flux of near-inertial waves. J. Phys. Oceanogr., 6, 894-908, https://doi.org/10.1175/ 1520-0485(1976)006<0894:OOTVPA > 2.0.CO;2.

MacKinnon, J. A., and K. Winters, 2005: Subtropical catastrophe: Significant loss of low-mode tidal energy at $28.9^{\circ}$. Geophys. Res. Lett., 32, L15605, https://doi.org/10.1029/2005GL023376.

- M. H. Alford, O. Sun, R. Pinkel, Z. Zhao, and J. Klymak, 2013a: The latitudinal dependence of shear and mixing in the Pacific transiting the critical latitude for PSI. J. Phys. Oceanogr., 43, 3-16, https://doi.org/10.1175/JPO-D-11-0107.1.

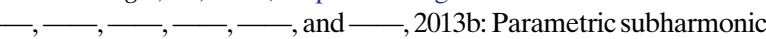
instability of the internal tide at $29^{\circ}$ N. J. Phys. Oceanogr., 43, 17-28, https://doi.org/10.1175/JPO-D-11-0108.1. 
— - and Coauthors, 2017: Climate Process Team on internal wave-driven ocean mixing. Bull. Amer. Meteor. Soc., 98, 2429-2454, https://doi.org/10.1175/BAMS-D-16-0030.1.

McComas, C. H., and F. P. Bretherton, 1977: Resonant interactions of oceanic internal waves. J. Geophys. Res., 82, 1397-1412, https://doi.org/10.1029/JC082i009p01397.

Metzger, E. J., and Coauthors, 2014: US Navy operational global ocean and arctic ice prediction system. Oceanography, 27 (3), 32-43, https://doi.org/10.5670/oceanog.2014.66.

Müller, M., B. K. Arbic, J. G. Richman, J. F. Shriver, E. L. Kunze, R. B. Scott, A. J. Wallcraft, and L. Zamudio, 2015: Toward an internal gravity wave spectrum in global ocean models. Geophys. Res. Lett., 42, 3474-3481, https://doi.org/10.1002/ 2015 GL063365.

Munk, W., and C. Wunsch, 1998: Abyssal recipes II: Energetics of tidal and wind mixing. Deep-Sea Res. I, 45, 1977-2010, https:// doi.org/10.1016/S0967-0637(98)00070-3.

Nash, J. D., M. H. Alford, and E. Kunze, 2005: Estimating internal wave energy fluxes in the ocean. J. Atmos. Oceanic Technol., 22, 1551-1570, https://doi.org/10.1175/JTECH1784.1.

Nikias, C. L., and A. P. Petropulu, 1993: Higher-Order Spectral Analysis:A Non-Linear Signal Processing Framework. Prentice Hall, $528 \mathrm{pp}$.

Niwa, Y., and T. Hibiya, 2011: Estimation of baroclinic tide energy available for deep ocean mixing based on three-dimensional global numerical simulations. J. Oceanogr., 67, 493-502, https://doi.org/10.1007/s10872-011-0052-1.

Qiu, B., S. Chen, and G. S. Carter, 2012: Time-varying parametric subharmonic instability from repeat CTD surveys in the northwestern Pacific Ocean. J. Geophys. Res., 117, C09012, https://doi.org/10.1029/2012JC007882.

Richet, O., C. Muller, and J.-M. Chomaz, 2017: Impact of a mean current on the internal tide energy dissipation at the critical latitude. J. Phys. Oceanogr., 47, 1457-1472, https://doi.org/ 10.1175/JPO-D-16-0197.1.

Richman, J. G., B. K. Arbic, J. F. Shriver, E. J. Metzger, and A. J. Wallcraft, 2012: Inferring dynamics from the wavenumber spectra of an eddying global ocean model with embedded tides. J. Geophys. Res., 117, C12012, https://doi.org/10.1029/ 2012JC008364.

Savage, A. C., and Coauthors, 2017: Frequency content of sea surface height variability from internal gravity waves to mesoscale eddies. J. Geophys. Res. Oceans, 122, 2519-2538, https://doi.org/10.1002/2016JC012331.

Shriver, J. F., B. K. Arbic, J. G. Richman, R. D. Ray, E. J. Metzger, A. J. Wallcraft, and P. G. Timko, 2012: An evaluation of the barotropic and internal tides in a high resolution global ocean circulation model. J. Geophys. Res., 117, C10024, https://doi.org/10.1029/2012JC008170.

_ J. G. Richman, and B. K. Arbic, 2014: How stationary are the internal tides in a high resolution global ocean circulation model? J. Geophys. Res. Oceans, 119, 2769-2787, https:// doi.org/10.1002/2013JC009423.

Simmons, H. L., 2008: Spectral modification and geographic redistribution of the semi-diurnal internal tide. Ocean Modell., 21, 126-138, https://doi.org/10.1016/j.ocemod.2008.01.002.

- , R. W. Hallberg, and B. K. Arbic, 2004: Internal wave generation in a global baroclinic tide model. Deep-Sea Res. II, 51, 3043-3068, https://doi.org/10.1016/j.dsr2.2004.09.015.

St. Laurent, L., and H. L. Simmons, 2006: Estimates of power consumed by mixing in the ocean interior. J. Climate, 19, 48774890, https://doi.org/10.1175/JCLI3887.1.

Sun, O. M., 2010: Subharmonic energy transfer from the semidiurnal internal tide at Kaena Ridge. Ph.D. thesis, University of California, San Diego, 109 pp.

_- , and R. Pinkel, 2012: Energy transfer from high-shear, lowfrequency internal waves to high-frequency waves near Kaena Ridge, Hawaii. J. Phys. Oceanogr., 42, 1524-1547, https://doi.org/10.1175/JPO-D-11-0117.1.

—_, and —_, 2013: Subharmonic energy transfer from the semidiurnal internal tide to near-diurnal motions over Kaena Ridge, Hawaii. J. Phys. Oceanogr., 43, 766-789, https://doi.org/10.1175/JPO-D-12-0141.1.

Sutherland, B. R., 2013: The wave instability pathways to turbulence. J. Fluid Mech., 724, 1-4, https://doi.org/10.1017/jfm.2013.149.

Timko, P. G., B. K. Arbic, J. G. Richman, R. B. Scott, E. J. Metzger, and A. J. Wallcraft, 2012: Skill tests of three-dimensional tidal currents in a global ocean model: A look at the North Atlantic. J. Geophys. Res., 117, C08014, https://doi.org/10.1029/2011JC007617.

$-,-,-,-\longrightarrow, \ldots$, and,- 2013 : Skill testing a threedimensional global tide model to historical current meter records. J. Geophys. Res. Oceans, 118, 6914-6933, https:// doi.org/10.1002/2013JC009071.

Wallcraft, A. J., E. J. Metzger, and S. N. Carroll, 2009: Software design description for the Hybrid Coordinate Ocean Model (HYCOM) version 2.2. Naval Research Laboratory Rep. NRL/MR/7320-09-9166, 149 pp., http://www.dtic.mil/dtic/tr/ fulltext/u2/a494779.pdf.

Wunsch, C., and A. Gill, 1976: Observations of equatorially trapped waves in Pacific sea level variations. Deep-Sea Res. Oceanogr. Abstr., 23, 371-390, https://doi.org/10.1016/0011-7471(76)90835-4.

Xie, X., X. Shang, G. Chen, and D. Wang, 2016: Poleward propagation of parametric subharmonic instability-induced inertial waves. J. Geophys. Res. Oceans, 121, 1881-1895, https://doi.org/10.1002/2015JC011194.

Young, W. R., Y. K. Tang, and N. J. Balmforth, 2008: Near-inertial parametric subharmonic instability. J. Fluid Mech., 607, 25-49, https://doi.org/10.1017/S0022112008001742.

Zhao, Z., M. H. Alford, J. A. MacKinnon, and R. Pinkel, 2010: Long-range propagation of the semi-diurnal internal tide from the Hawaiian ridge. J. Phys. Oceanogr., 40, 713-736, https:// doi.org/10.1175/2009JPO4207.1. 